\title{
Perioperative visual loss in ocular and nonocular surgery
}

This article was published in the following Dove Press journal:

Clinical Ophthalmology

4 June 2010

Number of times this article has been viewed

\section{Kathleen T Berg \\ Andrew R Harrison \\ Michael S Lee \\ Department of Ophthalmology, University of Minnesota, Minneapolis, MN, USA}

\begin{abstract}
Incidence estimates for perioperative vision loss (POVL) after nonocular surgery range from $0.013 \%$ for all surgeries up to $0.2 \%$ following spine surgery. The most common neuro-ophthalmologic causes of POVL are the ischemic optic neuropathies (ION), either anterior (AION) or posterior (PION). We identified 111 case reports of AION following nonocular surgery in the literature, with most occurring after cardiac surgery, and 165 case reports of PION following nonocular surgery, with most occurring after spine surgery or radical neck dissection. There were an additional 526 cases of ION that did not specify if the diagnosis was AION or PION. We also identified 933 case reports of central retinal artery occlusion (CRAO), 33 cases of pituitary apoplexy, and 245 cases of cortical blindness following nonocular surgery. The incidence of POVL following ocular surgery appears to be much lower than that seen following nonocular surgery. We identified five cases in the literature of direct optic nerve trauma, 47 cases of AION, and five cases of PION following ocular surgery. The specific pathogenesis and risk factors underlying these neuro-ophthalmic complications remain unknown, and physicians should be alert to the potential for loss of vision in the postoperative period.
\end{abstract}

Keywords: perioperative, postoperative, vision loss, ocular surgery, nonocular surgery

\section{Introduction}

Vision loss is a rare, and devastating, complication that can be seen following both ocular and nonocular surgeries. The specific pathogenesis of perioperative vision loss (POVL) remains elusive in most cases, with much controversy surrounding patient and surgical risk factors. Many publications have speculated on causation. Unfortunately, given the rarity of POVL, these are based solely on retrospective data. We present a detailed review of the literature on the subject of vision loss following ocular and nonocular surgical procedures.

\section{Method of literature review}

We searched the National Library of Medicine's PubMed database with a subsequent review of the accompanying references (last accessed February 8, 2010). The major search words and word combinations included: posterior ischemic optic neuropathy; anterior ischemic optic neuropathy; central retinal artery occlusion; pituitary apoplexy; cortical blindness; optic nerve trauma; postoperative vision loss; postoperative blindness; perioperative vision loss; perioperative blindness; and ocular surgery. In addition, the citations from the above searches were also included. Cases from the non-English literature and cases prior to 1970 were not included. Cases with documented direct surgical trauma to orbital structures other than the optic nerve were not included.
Correspondence: Michael S Lee 420 Delaware St SE, MMC 493, Minneapolis, MN 55455, USA

$\mathrm{Tel}+\mathrm{I} 6126253553$

Fax $+\mid$ 6|2-626-3||

Email mikelee@umn.edu 
Cases attributed to steroid injection into orbital structures were also not included.

\section{Neuro-ophthalmologic causes of vision loss after nonocular surgery \\ Incidence and prevalence of POVL}

Vision loss occurring after nonocular surgery with general anesthesia typically results from: anterior ischemic optic neuropathy (AION); posterior ischemic optic neuropathy (PION); central retinal artery occlusion (CRAO); pituitary apoplexy; or cortical blindness. While studies of $400,000^{1}$ and $61,000^{2}$ patients undergoing general anesthesia have demonstrated a low incidence of POVL in noncardiac, nonspinal fusion procedures, the actual incidence, overall, remains unknown. However, the risk of POVL is believed to be highest following cardiac or spine surgeries. A recent review of 5.6 million patients from the National Inpatient Sample (NIS) found that the incidence of POVL to be 8.64/10,000 after cardiac procedures and 3.09/10,000 after spinal fusion, while the incidence was only $0.66 / 10,000$ after cholecystectomy, and just 0.12/10,000 after appendectomy. ${ }^{3}$ In a retrospective study of 501,342 noncardiac surgeries carried out at one institution, only four patients $(0.0008 \%)$ developed persistent vision loss not attributed to direct surgical trauma to optic or cerebral tissues. ${ }^{1}$ In a separate study at the same institution, 17 patients were found to have perioperative ION out of a total of 27,915 coronary artery bypass graft $(\mathrm{CABG})$ procedures $(0.06 \%) .{ }^{4}$ Similar studies of POVL following cardiac surgery have shown comparable findings, with the incidence rates ranging from $0.09 \%{ }^{5}$ to $0.113 \% .{ }^{6}$ Other studies suggest that the rate of POVL may be even higher after spine surgery. A study of over 225,000 surgeries at a single institution over a 15 -year period found three cases of POVL out of 3,351 spine surgeries ( $0.09 \%)$, a 50-fold higher rate compared with other surgeries in the study. ${ }^{7}$ Additional large-scale studies of POVL after spine surgery have demonstrated incidence rates varying from $0.094 \%{ }^{8}$ to $0.2 \%,{ }^{9}$ though the higher incidence seen in the latter study may be because it was carried out at three tertiary care specialty hospitals, which may have attracted more complex cases.

\section{Ischemic optic neuropathies}

The two most common types of POVL are AION and PION. ${ }^{10,11}$ In a recent study of 126,666 surgeries from a single institution, there were 17 cases of ischemic optic neuropathy (ION) $(0.013 \%): 8$ AION and 9 PION. ${ }^{12}$ The type of ION varies depending on the type of surgery performed, with AION occurring most often after cardiac surgery ${ }^{45}$ and PION occurring most often after prone-positioned spine surgery or radical neck dissection. ${ }^{10,13}$

In a recent analysis of $4,728,815$ spine surgeries from the NIS registry, the overall incidence of postoperative ION was found to be $0.006 \%{ }^{8}$ Other incidence estimates for ION following spine surgery range from $0.028 \%{ }^{14}$ to $0.12 \%{ }^{9}$

A review of 27,915 cardiac surgeries found the overall incidence of ION to be $0.06 \%$, with 12 cases of AION and five cases of PION, ${ }^{4}$ while another study of $9,701 \mathrm{CABG}$ procedures found an ION incidence of $0.11 \%$, with eight cases of AION and three cases of PION. ${ }^{6}$ A review of 602 CABG procedures found the incidence of AION to be $1.3 \%,{ }^{15}$ while a similar study of 7,685 CABG procedures found an ION incidence of $0.09 \% .^{5}$

When the incidence values from these large-scale reviews are compiled, the overall incidence of AION is $0.00079 \%$ $(1 / 126,666)$ after spine surgery and $0.024 \%(42 / 172,569)$ following cardiac surgery. There were no cases of AION following noncardiac, nonspine surgery in any of the largescale reviews. When the incidence values for PION are compiled, the overall incidence is $0.005 \%(7 / 140,768)$ after spine surgery, $0.0061 \%(10 / 164,282)$ following cardiac surgery, and $0.0032 \%(4 / 126,666)$ after nonspinal, noncardiac surgery.

\section{Diagnosis}

AION is likely caused by occlusion or relative hypoperfusion of the anterior optic nerve head by the posterior ciliary arteries. ${ }^{16}$ Typical presentation includes sudden painless vision loss and a visual field defect, most commonly inferior altitudinal in nature. Diffuse or segmental disc edema is observed on funduscopy at symptom onset, with the development of optic nerve atrophy after 4-8 weeks (Figure 1). ${ }^{17}$ Three forms of AION are recognized: perioperative, arteritic, and nonarteritic. ${ }^{18}$ The arteritic form of AION, associated with giant cell arteritis (GCA), often presents in the elderly with an elevated erythrocyte sedimentation rate (ESR) and C-reactive protein (CRP), and systemic symptoms such as weight loss and jaw claudication. It is important to note that inflammatory markers are often extremely elevated after general surgery. Because of this, in the absence of other features of GCA such as systemic symptoms or abnormal temporal arteries, it is not advisable to check ESR and CRP in a patient who awakes from surgery with visual loss. If these are checked, an elevated ESR alone is not enough to diagnose arteritic AION in the postoperative period. ${ }^{19}$ The 


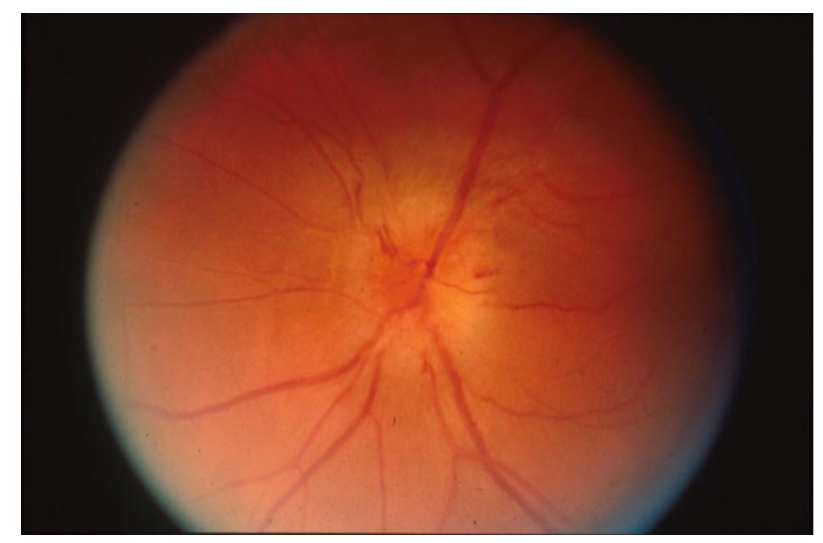

Figure I Optic disc edema observed in all cases of acute anterior ischemic optic neuropathy.

gold standard for diagnosing arteritic AION is the temporal artery biopsy. Giant cell arteritis is considered an ophthalmic emergency, requiring the urgent administration of systemic corticosteroids to prevent the development of permanent, bilateral blindness. ${ }^{18}$

PION is believed to result from infarction to the intraorbital optic nerve, supplied by pial blood vessels. PION is much less common than AION. There are three recognized types of PION: perioperative, arteritic, and nonarteritic; the perioperative type is the most common. ${ }^{20} \mathrm{PION}$ presents with similar signs and symptoms as AION, and is differentiated from AION based on the presence of a normal optic disc and fundoscopic examination at the onset of visual symptoms (Figure 2). ${ }^{13}$ Gadolinium enhancement ${ }^{21}$ and/or restricted diffusion ${ }^{22}$ may be seen on orbital magnetic resonance imaging (MRI), while the MRI is typically unremarkable in AION.

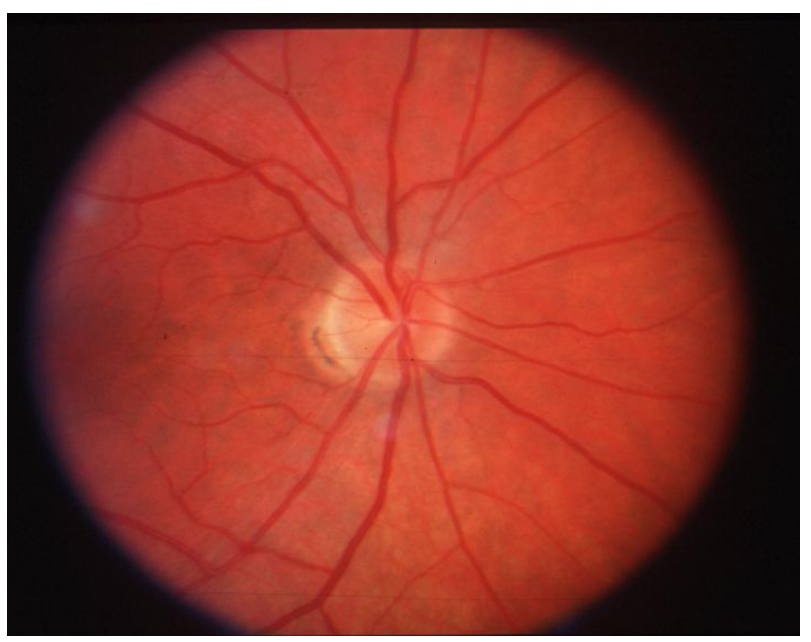

Figure 2 Normal appearing optic disc is seen in all cases of acute posterior ischemic optic neuropathy.

\section{Pathogenesis, risk factors, and outcomes}

The pathogenesis of postoperative ION remains undefined. AION may occur in the setting of substantial blood loss or hypotension, such as that seen following gastrointestinal bleeding or hemodialysis. It is for this reason that some have suggested that intraoperative hypotension and anemia underlie the development of ION. ${ }^{23}$ Of 83 cases of ION following spine surgery listed in the American Society of Anesthesiologists (ASA) Postoperative Visual Loss Registry, $96 \%$ had $1 \mathrm{~L}$ or more of blood loss or 6 hours or more of total anesthesia time, (with a mean anesthetic time of 9.6 hours). ${ }^{10}$ Blood loss, with or without arterial hypotension, has been shown to cause the release of endogenous vasoconstrictors due to the activation of the sympathetic nervous system, which can produce choroidal and optic nerve ischemia. ${ }^{24}$ The use of vasoconstricting agents to correct intraoperative hypotension has also been suggested to promote optic nerve ischemia. ${ }^{5,11,24}$ Analysis of the NIS registry found that patients who developed ION were more likely to have hypotension, peripheral vascular disease, or anemia. ${ }^{8}$ However, there have been cases of ION development in patients with blood pressure readings $>90 \mathrm{mmHg}^{24}$ and in those with minimal (less than $500 \mathrm{~mL}$ ) blood loss. ${ }^{10,25,26}$

Postoperative AION occurs most often after cardiac surgery, especially CABG. The mild to moderate hypothermia used during bypass could cause increased blood viscosity, which may lead to watershed infarction of the optic nerve. Indeed, it has been shown that cerebral blood flow decreases by $6 \%-7 \%$ for every degree Centigrade drop in body temperature ${ }^{27}$ Cardiopulmonary bypass has also been shown to promote complement activation, causing a five-fold increase in $\mathrm{C} 3 \mathrm{a}$ levels, a known smooth muscle spasminogen. ${ }^{28}$ A recent time-matched, case-control study found that patients who developed AION after CABG had lower postoperative hemoglobin values, a higher rate of atherosclerotic disease, and were more likely to have had a coronary angiogram within 48 hours of surgery when compared with control CABG patients. ${ }^{4} \mathrm{~A}$ similar study found that $\mathrm{CABG}$ patients who developed postoperative AION had prolonged bypass times, lower hematocrit levels, marked perioperative weight gain from large fluid infusions required to support intraoperative blood pressure, and lower cardiac outputs requiring higher doses of inotropic medications. ${ }^{15}$

CABG could also contribute to the development of AION through elevations in intraocular pressure (IOP). It has been noted that AION has occurred with elevations in $\mathrm{IOP}^{29,30}$ and there is experimental evidence that elevated IOP can cause optic nerve ischemia by reducing blood flow to the eye. ${ }^{11}$ 
The perfusion pressure of the eye is equal to the mean arterial pressure (MAP) minus the IOP, and dramatic changes in perfusion pressure may overwhelm the autoregulation of blood flow to the eye. ${ }^{18}$ It has been shown that there is a rapid rise in IOP lasting about 30 minutes when bypass pump circulation begins, ${ }^{31}$ and IOP may remain elevated for as long as three days after bypass. ${ }^{32}$ The IOP elevation seen in CABG is believed to be due in part to hemodilution, which causes an increase in intraocular blood flow along with a decrease in colloidal osmotic pressure. ${ }^{31}$ Autoregulation to the eye may also be disrupted by other systemic processes often seen in patients who are candidates for $\mathrm{CABG}$, such as aging and diabetes mellitus, as well as marked arterial hypotension, ${ }^{33}$ which often occurs during bypass procedures. Heart disease patients also often have chronic hypertension, which may reduce tolerance to periods of hypotension by shifting the autoregulatory curve of optic nerve blood flow. ${ }^{34}$

A variety of hemodynamic derangements could conceivably cause PION including: anemia, hypotension, increased venous pressure, prone positioning during surgery, increased cerebrospinal fluid pressure, direct ocular compression, and embolism. Alteration is usually seen in more than one of these parameters in a given patient, suggesting that the mechanism of infarction may be multifactorial. Anemia and hypotension are nearly always seen in patients who develop postoperative PION. ${ }^{13}$ The pial vessels that supply the posterior optic nerve lack an autoregulatory mechanism, and are therefore prone to ischemia during periods of systemic hypotension and when the blood oxygen carrying capacity is decreased. ${ }^{35}$ However, studies that compared patients with POVL after spine surgery to controls found no difference in perioperative hematocrit and blood pressure, suggesting that hypotension and anemia alone are not enough to precipitate PION. ${ }^{12,36}$

Postoperative PION is most commonly seen following spine surgery in the prone position. Prone positioning may lead to increased orbital venous pressure through an increase in abdominal venous pressure, especially when patients are obese. ${ }^{37}$ Spine surgery patients are often placed in the Trendelenburg position, which also results in greater orbital venous pressure (Figure 3). Prone positioning may exert excessive pressure on the globe, and has been shown to raise the IOP in both anesthetized ${ }^{38}$ and awake ${ }^{39}$ patients. The use of a Mayfield headrest can also exert excess pressure on the globe, especially in patients with exophthalmos or a flattened nasal bridge (Figure 4$).{ }^{40}$ However, an elevated IOP would be expected to decrease perfusion pressure to the optic nerve head and central retinal artery, resulting in AION or CRAO, rather than PION. ${ }^{13}$ However, avoidance of the

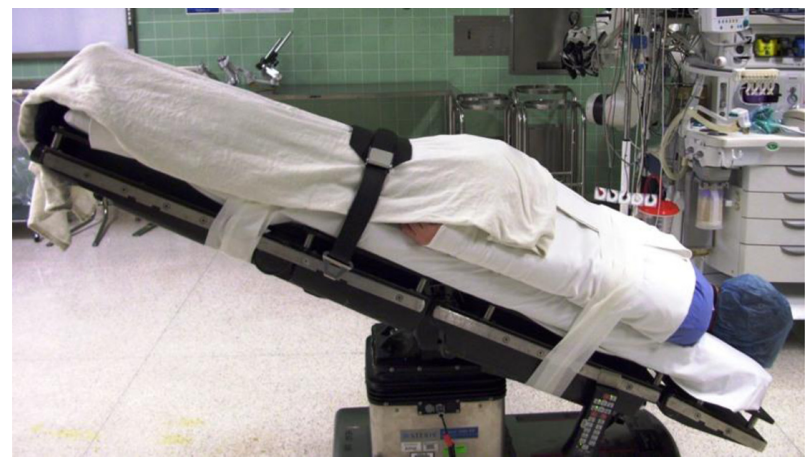

Figure 3 Positioning in spine surgery typically involves the patient lying prone in the Trendelenburg position. The level of the head is below the level of the feet, which leads to facial edema.

prone position and Mayfield headrests alone appears to be insufficient in preventing postoperative PION, as PION has been documented following surgery in the supine position, with the use of head pins instead of foam cushions in the prone position, ${ }^{10,41}$ and in the lateral decubitus position. ${ }^{42}$

A study of 37 patients with POVL (including 14 PION and 8 AION patients) following spine surgery found that patients were more likely to have surgical times greater than 6.5 hours and substantial blood loss (3.5 L or more) when compared to age- and surgery-matched controls. Perioperative hematocrit and blood pressure did not differ between cases and controls. This study has been recognized as one of the few comparing hemodynamic factors between cases and controls. However, this study was poorly designed. It included

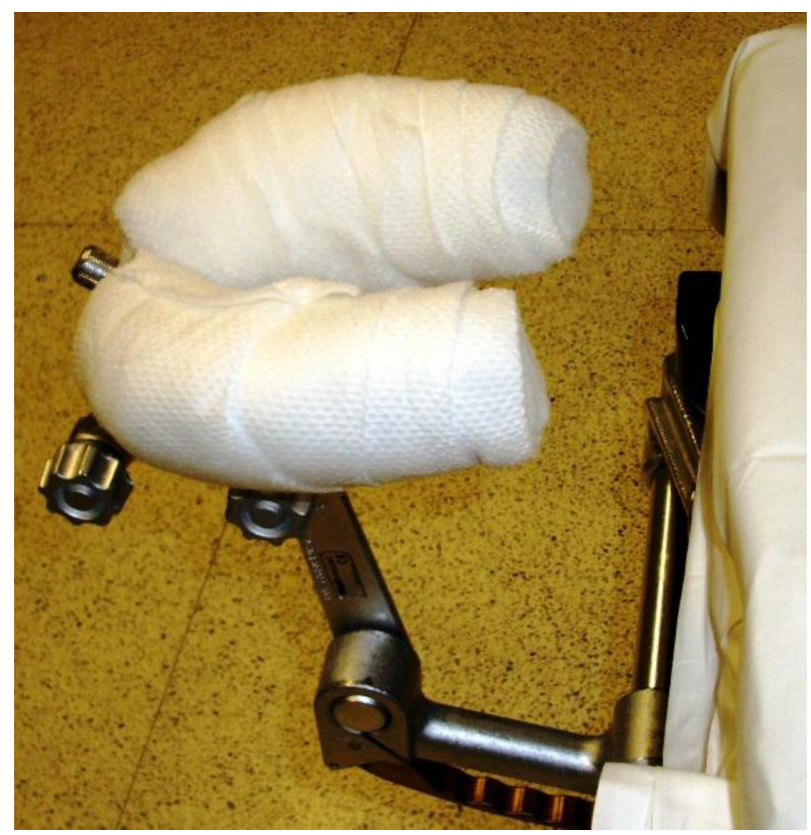

Figure 4 Mayfield headrest used in spine surgery. The edge of the headrest may compress the globe and lead to central retinal artery occlusion. 
10 patients from pre-existing case reports and the rest were cases from the authors' institution or data received from a solicitation to a spine society. The controls were taken from the authors' institution rather than matched to where the surgery took place. Finally, all of the hemodynamic variables were not available for all of the cases. ${ }^{36}$ Meanwhile, Holy and colleagues identified 17 patients with ION from a single institution. Each case was assigned two control patients, matched for age and gender, who underwent similar surgeries at the same institution with a similar date of surgery. All preoperative, intraoperative, and postoperative hemodynamic variables were available for cases and controls. There was no significant difference between cases and controls in any single hemodynamic variable, suggesting that the cause of ION following nonocular surgery is multifactorial. Almost all patients who develop postoperative ION experience some degree of blood loss, anemia, and hypotension, though not to a greater degree than that seen in control patients who did not suffer ION after surgery. ${ }^{12}$

There have been many documented cases of PION following radical neck dissection. Distension of ophthalmic veins, with accompanying facial edema, due to internal jugular vein ligation has been proposed as a potential mechanism for PION development. The venous distention could cause compression of the orbital apex, resulting in reduced perfusion and further anoxia of the posterior aspect of the optic nerve. $^{23,43}$

While there is no established treatment for postoperative AION or PION, some have documented an improvement in visual acuity after the correction of anemia with blood transfusion, ${ }^{9,44}$ the correction of hypotension with vasopressors, ${ }^{45}$ or the use of high-dose corticosteroids. ${ }^{9}$ However, some patients recover full acuity without any treatment, ${ }^{46}$ suggesting that the improvements seen following the proposed interventions may be merely coincidental.

\section{Anterior ischemic optic neuropathy literature review}

Through our review of the literature, we encountered 111 reports of AION following nonocular surgery, as well as an additional 526 reports defined only as "ischemic optic neuropathy" (Table 1)..$^{8,10,47,48}$ While AION was most commonly seen after: cardiac procedures; ${ }^{4-6,12,15,19,47,49-57}$ it also occurred after spine surgery; ${ }^{9,10,12,36,58-63}$ shoulder surgery; ${ }^{64,65}$ radical neck dissection; ${ }^{66-70}$ total parathyroidectomy; ${ }^{55}$ peripheral vascular surgery $;^{71}$ emergent Cesarian section; ${ }^{44}$ transurethral prostatectomy $;{ }^{72}$ radical prostatectomy $;{ }^{73}$ hip replacement; $;{ }^{74}$ osteosynthesis; ${ }^{74}$ bilateral knee replacement; ${ }^{74}$
Table I Ischemic optic neuropathy cases following nonocular surgery

\begin{tabular}{|c|c|c|c|}
\hline $\begin{array}{l}\text { Number of } \\
\text { patients, } \\
\text { reference }\end{array}$ & Age; gender & $\begin{array}{l}\text { Surgery } \\
\text { performed }\end{array}$ & Diagnosis \\
\hline${ }^{\mathrm{a}} 271^{8}$ & $\mathrm{NA} ; 5 \mathrm{I} \%$ male & Spine surgery & $\begin{array}{l}\text { ION, not } \\
\text { further } \\
\text { specified }\end{array}$ \\
\hline $245^{3}$ & NA & $\begin{array}{l}\text { Spinal, orthopedic, } \\
\text { Cardiac, and general } \\
\text { Surgery }\end{array}$ & $\begin{array}{l}\text { ION, not } \\
\text { further } \\
\text { specified }\end{array}$ \\
\hline $8^{10}$ & $36-64 ; 72 \%$ male & Spine surgery & $\begin{array}{l}\text { ION, not } \\
\text { further } \\
\text { specified }\end{array}$ \\
\hline $2^{47}$ & NA & CABG & $\begin{array}{l}\text { ION, not } \\
\text { further } \\
\text { specified }\end{array}$ \\
\hline $19^{10}$ & $36-64 ; 72 \%$ male & Spine surgery & AION \\
\hline a $8^{36}$ & I2-68; gender NA & Spine surgery & AION \\
\hline $1^{62}$ & $65 \mathrm{~F}$ & Spine surgery & AION \\
\hline $1^{62}$ & $60 \mathrm{~F}$ & Spine surgery & AION \\
\hline $1^{62}$ & $4 \mid M$ & Spine surgery & AION \\
\hline $1^{58}$ & $57 \mathrm{M}$ & Spine surgery & AION \\
\hline $1^{59}$ & $50 \mathrm{~F}$ & Spine surgery & AION \\
\hline $1^{60}$ & $16 \mathrm{~F}$ & Spine surgery & AION \\
\hline $1^{61}$ & $44 M$ & Spine surgery & AION \\
\hline $1^{9}$ & $56 \mathrm{~F}$ & Spine surgery & AION \\
\hline $1^{12}$ & $71 \mathrm{M}$ & Spine surgery & AION \\
\hline $12^{4}$ & $57-73 ; 71 \%$ male & CABG & AION \\
\hline $8^{15}$ & $58-70 ; 63 \%$ male & CABG & AION \\
\hline $8^{6}$ & 39-8I; 82\% male & CABG & AION \\
\hline $7^{5}$ & 44-6I; all male & CABG & AION \\
\hline $3^{63}$ & NA & CABG & AION \\
\hline $1^{12}$ & $60 M$ & CABG & AION \\
\hline $1^{12}$ & $54 M$ & CABG & AION \\
\hline $1^{12}$ & $64 M$ & CABG & AION \\
\hline $1^{12}$ & $55 M$ & CABG & AION \\
\hline $1^{12}$ & $72 M$ & CABG & AION \\
\hline $1^{12}$ & $60 M$ & CABG & AION \\
\hline $1^{12}$ & $54 \mathrm{M}$ & CABG & AION \\
\hline $1^{19}$ & $54 M$ & CABG & AION \\
\hline $1^{19}$ & $54 \mathrm{M}$ & CABG & AION \\
\hline $1^{19}$ & $63 M$ & CABG & AION \\
\hline $1^{31}$ & $46 M$ & CABG & AION \\
\hline $1^{31}$ & $63 \mathrm{M}$ & CABG & AION \\
\hline $1^{30}$ & $55 \mathrm{M}$ & CABG & AION \\
\hline $1^{30}$ & $68 M$ & CABG & AION \\
\hline $1^{71}$ & $68 M$ & CABG & AION \\
\hline $1^{71}$ & $74 M$ & CABG & AION \\
\hline $1^{55}$ & $56 M$ & CABG & AION \\
\hline $1^{55}$ & $44 M$ & CABG & AION \\
\hline $1^{56}$ & $71 \mathrm{~F}$ & CABG & AION \\
\hline $1^{56}$ & $58 \mathrm{M}$ & CABG & AION \\
\hline $1^{57}$ & $63 M$ & CABG & AION \\
\hline $1^{51}$ & $71 \mathrm{~F}$ & CABG & AION \\
\hline $1^{53}$ & $70 M$ & $\mathrm{CABG}^{53}$ & AION \\
\hline $1^{54}$ & $63 \mathrm{M}$ & CABG & AION \\
\hline $1^{67}$ & $47 M$ & Neck dissection & AION \\
\hline $1^{69}$ & $48 M$ & Neck dissection & AION \\
\hline
\end{tabular}


Table I (Continued)

\begin{tabular}{|c|c|c|c|}
\hline $\begin{array}{l}\text { Number of } \\
\text { patients, } \\
\text { reference }\end{array}$ & Age; gender & $\begin{array}{l}\text { Surgery } \\
\text { performed }\end{array}$ & Diagnosis \\
\hline$\overline{1^{70}}$ & $60 M$ & Neck dissection & $\mathrm{AION}$ \\
\hline $1^{55}$ & $16 \mathrm{~F}$ & $\begin{array}{l}\text { Total } \\
\text { parathyroidectomy }\end{array}$ & AION \\
\hline 149 & $40 M$ & $\begin{array}{l}\text { Dynamic } \\
\text { cardiomyoplasty }\end{array}$ & AION \\
\hline $1^{52}$ & $64 \mathrm{~F}$ & $\begin{array}{l}\text { Coronary } \\
\text { catheterization }\end{array}$ & $\mathrm{AION}$ \\
\hline $1^{71}$ & $62 \mathrm{~F}$ & $\begin{array}{l}\text { Peripheral vascular } \\
\text { surgery }\end{array}$ & AION \\
\hline $1^{64}$ & $45 \mathrm{M}$ & Shoulder surgery & AION \\
\hline $1^{65}$ & $55 M$ & Shoulder surgery & AION \\
\hline $1^{74}$ & $52 M$ & Hip replacement & AION \\
\hline $1^{74}$ & $75 M$ & Hip replacement & AION \\
\hline $1^{74}$ & $80 \mathrm{~F}$ & Hip replacement & AION \\
\hline $1^{74}$ & $55 M$ & Osteosynthesis & AION \\
\hline $1^{74}$ & $66 \mathrm{M}$ & $\begin{array}{l}\text { Bilateral knee } \\
\text { replacement }\end{array}$ & $\mathrm{AION}$ \\
\hline $1^{44}$ & $29 \mathrm{~F}$ & Cesarian section & AION \\
\hline$I^{72}$ & $66 M$ & $\begin{array}{l}\text { Transurethral } \\
\text { prostatectomy }\end{array}$ & AION \\
\hline $1^{73}$ & $50 M$ & $\begin{array}{l}\text { Radical } \\
\text { prostatectomy }\end{array}$ & AION \\
\hline $1^{75}$ & $47 \mathrm{~F}$ & Liposuction & AION \\
\hline $56^{10}$ & $36-64 ; 72 \%$ male & Spine surgery & PION \\
\hline $14^{20}$ & $\begin{array}{l}\text { mean age } 43.3 ; \\
\text { gender NA }\end{array}$ & Spine surgery & PION \\
\hline al $4^{36}$ & I2-68; gender NA & Spine surgery & PION \\
\hline $1^{37}$ & $66 \mathrm{M}$ & Spine surgery & PION \\
\hline $1^{37}$ & $43 \mathrm{M}$ & Spine surgery & PION \\
\hline $1^{37}$ & $12 M$ & Spine surgery & PION \\
\hline $1^{37}$ & $57 \mathrm{M}$ & Spine surgery & PION \\
\hline $1^{37}$ & $44 \mathrm{M}$ & Spine surgery & PION \\
\hline$I^{14}$ & I3; gender NA & Spine surgery & PION \\
\hline $1^{14}$ & I3; gender NA & Spine surgery & PION \\
\hline$I^{14}$ & 43; gender NA & Spine surgery & PION \\
\hline $1^{14}$ & 29; gender NA & Spine surgery & PION \\
\hline $1^{12}$ & $63 \mathrm{M}$ & Spine surgery & PION \\
\hline $1^{12}$ & $77 \mathrm{M}$ & Spine surgery & PION \\
\hline $1^{12}$ & $66 M$ & Spine surgery & PION \\
\hline $1^{62}$ & $49 \mathrm{~F}$ & Spine surgery & PION \\
\hline $1^{9}$ & $79 \mathrm{~F}$ & Spine surgery & PION \\
\hline $1^{9}$ & $27 \mathrm{~F}$ & Spine surgery & PION \\
\hline $1^{9}$ & $37 M$ & Spine surgery & PION \\
\hline $1^{77}$ & $24 M$ & Spine surgery & PION \\
\hline $1^{77}$ & $44 M$ & Spine surgery & PION \\
\hline $1^{78}$ & $33 \mathrm{~F}$ & Spine surgery & PION \\
\hline $1^{79}$ & $24 M$ & Spine surgery & PION \\
\hline $1^{71}$ & $13 M$ & Spine surgery & PION \\
\hline $1^{80}$ & $51 \mathrm{M}$ & Spine surgery & PION \\
\hline $1^{42}$ & $59 M$ & Spine surgery & PION \\
\hline $1^{81}$ & $48 M$ & Spine surgery & PION \\
\hline $1^{46}$ & $58 M$ & Spine surgery & PION \\
\hline $1^{82}$ & $68 \mathrm{~F}$ & Spine surgery & PION \\
\hline $1^{60}$ & $10 M$ & Spine surgery & PION \\
\hline$\left.\right|^{83}$ & $60 M$ & Spine surgery & PION \\
\hline
\end{tabular}

Table I (Continued)

\begin{tabular}{|c|c|c|c|}
\hline $\begin{array}{l}\text { Number of } \\
\text { patients, } \\
\text { reference }\end{array}$ & Age, gender & $\begin{array}{l}\text { Surgery } \\
\text { performed }\end{array}$ & Diagnosis \\
\hline 143,181 & $67 \mathrm{M}$ & Neck dissection & PION \\
\hline$\left.\right|^{84}$ & $71 M$ & Neck dissection & PION \\
\hline $1^{85}$ & $48 M$ & Neck dissection & PION \\
\hline $1^{86}$ & $49 M$ & Neck dissection & PION \\
\hline $1^{68}$ & $64 M$ & Neck dissection & PION \\
\hline$I^{182}$ & $73 M$ & Neck dissection & PION \\
\hline $1^{87}$ & $37 M$ & Neck dissection & PION \\
\hline $\mathrm{I}^{\prime}$ & $77 M$ & Neck dissection & PION \\
\hline $\mathrm{I}^{\prime}$ & $67 \mathrm{~F}$ & Neck dissection & PION \\
\hline $5^{4}$ & $57-73 ; 71 \%$ male & CABG & PION \\
\hline $3^{6}$ & $39-8 I ; 82 \%$ male & CABG & PION \\
\hline $1^{12}$ & $61 \mathrm{M}$ & CABG & PION \\
\hline $1^{12}$ & $68 \mathrm{~F}$ & CABG & PION \\
\hline $1^{21}$ & $57 \mathrm{~F}$ & CABG & PION \\
\hline$\left.\right|^{88}$ & $46 \mathrm{~F}$ & CABG & PION \\
\hline $1^{71}$ & $81 \mathrm{~F}$ & CABG & PION \\
\hline $3^{91}$ & $59 M$ & Rhinoplasty & PION \\
\hline 191 & $40 M$ & Rhinoplasty & PION \\
\hline $1^{91}$ & $23 \mathrm{~F}$ & Rhinoplasty & PION \\
\hline $1^{88}$ & $62 M$ & $\begin{array}{l}\text { Drainage of infected } \\
\text { hip prosthesis }\end{array}$ & PION \\
\hline $1^{89}$ & $64 M$ & Shoulder surgery & PION \\
\hline $1^{37}$ & $65 \mathrm{~F}$ & Knee surgery & PION \\
\hline $1^{12}$ & $66 \mathrm{M}$ & Knee surgery & PION \\
\hline $1^{12}$ & $62 M$ & Osteosynthesis & PION \\
\hline $1^{74}$ & $51 M$ & Osteosynthesis & PION \\
\hline $1^{23}$ & $59 \mathrm{~F}$ & Gastroduodenotomy & PION \\
\hline $1^{71}$ & $51 \mathrm{~F}$ & Abdominal exploration & nPION \\
\hline $\mathrm{I}^{\prime}$ & $52 M$ & Thoracotomy & PION \\
\hline $1^{12}$ & $88 M$ & $\begin{array}{l}\text { Thoracotomy and } \\
\text { segmentectomy }\end{array}$ & PION \\
\hline $1^{25}$ & $43 \mathrm{~F}$ & $\begin{array}{l}\text { Breast augmentation } \\
\text { and abdominal } \\
\text { liposuction }\end{array}$ & PION \\
\hline $1^{90}$ & $4 \mid M$ & Axillary vein grafting & PION \\
\hline $1^{26}$ & $42 M$ & $\begin{array}{l}\text { Laparoscopic } \\
\text { nephrectomy }\end{array}$ & PION \\
\hline $1^{12}$ & $70 M$ & $\begin{array}{l}\text { Femoral aneurysm } \\
\text { repair }\end{array}$ & PION \\
\hline $14^{20}$ & $\begin{array}{l}\text { mean age } 58.3 \text {; } \\
\text { gender NA }\end{array}$ & NA & PION \\
\hline
\end{tabular}

Notes: aStudy contains information compiled from data submitted to a registry, or compiled case reports, which may be redundant with that seen in the other papers listed.

Abbreviations: ION, ischemic optic neuropathy; AION, anterior ischemic optic neuropathy; PION, posterior ischemic optic neuropathy; NA, information not available; $M$, male; $F$, female; CABG, cardiopulmonary bypass grafting.

and liposuction. ${ }^{75,76}$ Patients ranged in age from $16^{55,60}$ to 80 years. ${ }^{74}$ Of the 75 cases that provided individual patient information, 43 cases were bilateral (57\%). Of the 32 cases in which the information was provided for individual patients, 27 (84\%) reported perioperative "anemia", which we defined as a hemoglobin of less than $10 \mathrm{gm} / \mathrm{dL}$ and/or a hematocrit less than $30 \%$. Of the 38 cases that provided 
perioperative blood pressure measurements for individual patients, 18 (48\%) reported perioperative systolic blood pressure (SBP) measurements of less than $90 \mathrm{mmHg}$ and/ or MAP measurements less than 70 .

\section{Posterior ischemic optic neuropathy literature review}

We are aware of 165 cases of postoperative PION in the literature, as well as an additional 526 reports defined only as "ischemic optic neuropathy" (Table 1)..$^{8,10,47,48}$ The most common procedure that resulted in PION was spine surgery. . $^{9} 10,12,14,20,36,37,42,46,60,62,71,77-83$ PION has also been documented following: radical neck dissection; ${ }^{1,43,68,84-87}$ CABG; $;^{4,6,21,71,88}$ knee surgery; ${ }^{12,37}$ osteosynthesis; ${ }^{12,74}$ drainage of an infected hip prosthesis ${ }^{88}$ shoulder surgery; ${ }^{89}$ gastroduodenotomy; ${ }^{23}$ abdominal exploration; ${ }^{71}$ thoracotomy; ${ }^{1,12}$ axillary vein grafting $;{ }^{90}$ femoral aneurysm repair; ${ }^{12}$ laparoscopic nephrectomy; ${ }^{26}$ rhinoplasty $;{ }^{91}$ and breast augmentation with abdominal liposuction. ${ }^{25}$ Patients ranged in age from $10^{60}$ to $81 .{ }^{71}$ Of the 80 cases that provided individual patient data, 47 (59\%) were bilateral. Of the 42 cases in which the information was provided for individual patients, 28 (67\%) reported perioperative "anemia", which we defined as hemoglobin of less than $10 \mathrm{gm} / \mathrm{dL}$ and/or hematocrit less than $30 \%$. Of the 42 cases that provided blood pressure measurements, $28(67 \%)$ reported perioperative SBP readings of less than $90 \mathrm{mmHg}$ and/or MAP measurements less than 70 .

\section{Central retinal artery occlusion}

Central retinal artery occlusion (CRAO) has been welldocumented in children and adults following trauma, ${ }^{92}$ and embolic, ${ }^{93}$ thrombotic, or vasospastic episodes. ${ }^{94}$ Of the 93 spine surgery cases submitted to the ASA Postoperative Visual Loss Registry, there were 10 cases of CRAO, 7 of which resulted in acuity readings of no light perception. ${ }^{10}$ In a recent analysis of 4,728,815 spine surgeries from the NIS registry, the overall incidence of postoperative CRAO was found to be $0.001 \%{ }^{8}$

\section{Diagnosis}

CRAO presents with a cherry-red spot on the macula, a white ground-glass appearance of the retina, attenuated arterioles with preserved choriocapillaries, and an afferent pupillary defect (Figure 5). ${ }^{10}$

\section{Pathogenesis, risk factors, and outcomes}

CRAO occurring after general surgery is typically observed following external ocular compression. When compared with

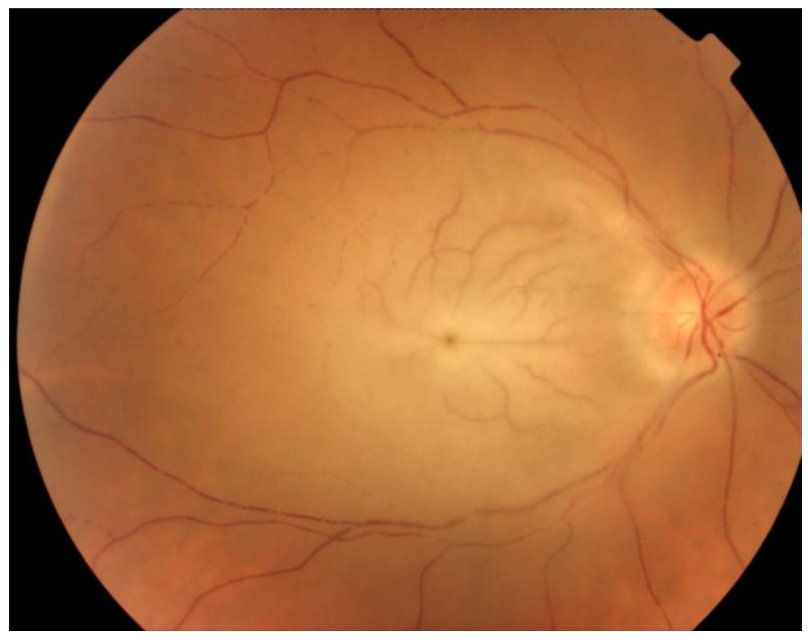

Figure 5 Funduscopic appearance of central retinal artery occlusion demonstrates a cherry-red spot on the macula, attenuated arterioles, and retinal whitening from edema.

cases of ION after spine surgery, patients who developed CRAO were found to have significantly lower mean anesthetic durations and median estimated blood losses. Periocular trauma was documented in the majority (70\%) of cases, evidenced by ipsilateral decreased supraorbital sensation, ophthalmoplegia, corneal abrasion, ptosis, or unilateral erythema. ${ }^{10}$ The ophthalmic artery gives rise to the central retinal artery, as well as the posterior ciliary artery, which supplies the choroid. Extensive pressure on the globe may raise IOP above the SBP leading to retinal ischemia. ${ }^{95}$ Experiments in rhesus monkeys have shown that the retina can tolerate pressure-induced ischemia for approximately 95 minutes and still recover, although it suffers permanent ischemic damage after 105 minutes. ${ }^{96}$ The majority of postoperative CRAO cases occur in prone-positioned patients, or in those positioned supine with excessive ocular pressure secondary to an anesthetic mask. ${ }^{95}$

No Class I data on the effective treatment for CRAO exists, and the accompanying blindness is often permanent. It has been suggested that the use of a foam headrest with orbital cutouts may prevent postoperative CRAO development. ${ }^{95}$

\section{CRAO literature review}

We are aware of 933 documented cases of postoperative CRAO (Table 2), with the overwhelming majority seen following spinal surgery. , $10,40,48,95,97-104$ Cases were also documented after hip/femur treatment, ${ }^{33}$ knee replacement, ${ }^{3}$ cholecystectomy, ${ }^{3}$ appendectomy, ${ }^{3}$ colorectal resection, ${ }^{3}$ and cardiac surgery. ${ }^{3}$ There was also one case following hip replacement in the lateral decubitus position without direct pressure on the orbit. The authors speculate that the CRAO 
Table 2 Central retinal artery occlusion case reports

\begin{tabular}{|c|c|c|}
\hline Number of patients, reference & Age, gender & Surgery performed \\
\hline${ }^{2} 86^{43}$ & NA & Spinal, orthopedic, cardiac, and general surgery \\
\hline${ }^{\mathrm{b}} 47^{8}$ & NA & Spine surgery \\
\hline $\mathrm{b} 10^{10}$ & 33-59; gender NA & Spine surgery \\
\hline 197 & $12 \mathrm{~F}$ & Spine surgery \\
\hline$I^{40}$ & $28 \mathrm{~F}$ & Spine surgery \\
\hline $1^{98}$ & $30 M$ & Spine surgery \\
\hline $1^{99}$ & $23 M$ & Spine surgery \\
\hline 100 & NA & Spine surgery \\
\hline $1^{101}$ & $29 M$ & Spine surgery \\
\hline$I^{102}$ & $12 \mathrm{~F}$ & Spine surgery \\
\hline $1^{103}$ & $73 \mathrm{M}$ & Spine surgery \\
\hline 104 & $38 M$ & Spine surgery \\
\hline 195 & $12 \mathrm{~F}$ & Spine surgery \\
\hline $1^{105}$ & $58 M$ & Hip replacement \\
\hline
\end{tabular}

Notes: a Study results include all causes of retinal vascular occlusion, not exclusively CRAO; 'btudy contains information compiled from data submitted to registry, or compiled case reports, which may be redundant with that seen in other papers listed.

Abbreviations: NA, information not available; $M$, male; $F$, female.

in this case may have been due to venous hypertension from a chest strap, which could have resulted in IOP elevation. ${ }^{105}$ All cases were unilateral, and most authors reported postoperative periocular swelling and/or erythema. Patients ranged in age from $12^{95,97,102}$ to 73 years. ${ }^{103}$

\section{Pituitary apoplexy Diagnosis}

Pituitary apoplexy is a rare clinical syndrome caused by infarction or hemorrhage of an existing pituitary adenoma. There is typically rapid enlargement of the adenoma, leading to compression of parasellar structures and the abrupt onset of signs and symptoms such as headache, meningismus, vomiting, visual field defects, unilateral or bilateral vision loss, ophthalmoplegia, and/or stupor. ${ }^{106} \mathrm{MRI}$ is the imaging modality of choice in the diagnosis of pituitary apoplexy, with a sensitivity of $88 \%$ (Figure 6 ). ${ }^{107}$

\section{Pathogenesis, risk factors, and outcomes}

Autopsy studies have suggested that the overall incidence of pituitary adenomas in adults is $1.4 \%$, with the vast majority being completely asymptomatic. ${ }^{108}$ There are many factors implicated in the development of pituitary apoplexy, including major surgery. ${ }^{109}$ During surgery, fluctuations in blood pressure, hypotension, blood dilution with crystalloid, anticoagulation, excessive steroid secretion, and transient increases in intracranial pressure may all play a role in pituitary apoplexy pathogenesis. ${ }^{110}$ Postoperative pituitary apoplexy occurs most often after cardiac surgery, ${ }^{110}$ especially CABG. ${ }^{111}$ In fact, an autopsy study of patients who died within 10 days of CABG found that $15 \%$ had evidence of pituitary necrosis, suggesting that pituitary apoplexy following CABG may be even more common than its clinical recognition. ${ }^{108}$ Factors specifically associated with bypass that may lead to pituitary apoplexy include reduced tissue oxygenation, embolism, anticoagulation, edema, and/or positive pressure ventilation. ${ }^{111}$

The recommended management for pituitary apoplexy is urgent transsphenoidal decompression surgery and highdose corticosteroid administration. ${ }^{112}$ However, a more

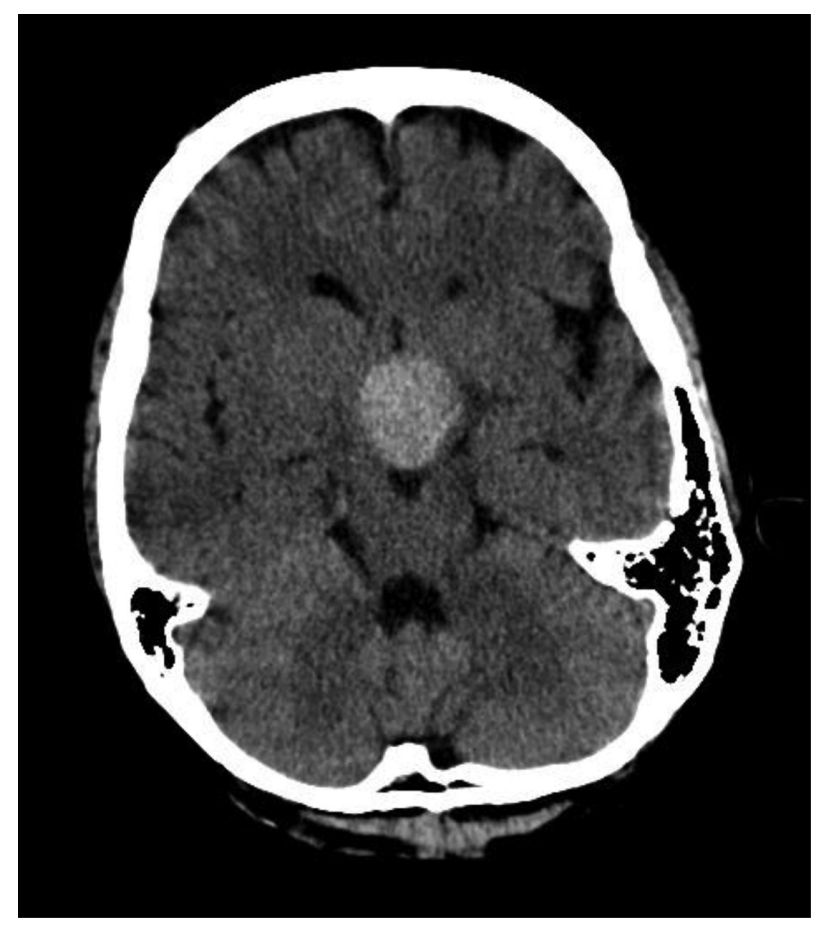

Figure 6 Head computed tomography of a patient with pituitary apoplexy. The hyperdense region represents hemorrhage within a pre-existing pituitary adenoma. 
conservative medical approach can be considered when vision is unaffected, or when the visual deficit is stable or improving, because spontaneous recovery may ensue. ${ }^{113}$

\section{Pituitary apoplexy literature review}

We are aware of 33 cases in the literature of postoperative pituitary apoplexy (Table 3). The majority of cases occurred following cardiac surgery, especially CABG,,${ }^{111,114-124}$ as well as mitral valve repair, ${ }^{125}$ and aortic valve replacement. ${ }^{126}$ Pituitary apoplexy was also seen following: abdominal aortic aneurysm repair; ${ }^{127}$ liposuction; ${ }^{128}$ knee arthroplasty; ${ }^{129,130}$ hip arthroplasty; ${ }^{114,130}$ spine surgery; ${ }^{131}$ cholecystectomy; ${ }^{132}$

Table 3 Pituitary apoplexy case reports

\begin{tabular}{|c|c|c|}
\hline $\begin{array}{l}\text { Number of patients, } \\
\text { reference }\end{array}$ & Age, gender & Surgery performed \\
\hline$I^{114}$ & $69 \mathrm{~F}$ & CABG \\
\hline$\left.\right|^{114}$ & $65 \mathrm{M}$ & CABG \\
\hline $1^{116}$ & $57 M$ & CABG \\
\hline$\left.\right|^{116}$ & $55 \mathrm{M}$ & CABG \\
\hline$\left.\right|^{118}$ & $58 M$ & CABG \\
\hline $1^{118}$ & $65 M$ & CABG \\
\hline $1^{118}$ & $68 M$ & CABG \\
\hline $1^{122}$ & $72 M$ & CABG \\
\hline$\left.\right|^{122}$ & $64 M$ & CABG \\
\hline $1^{123}$ & $79 M$ & CABG \\
\hline$\left.\right|^{123}$ & $64 M$ & CABG \\
\hline$\left.\right|^{183}$ & $77 \mathrm{M}$ & CABG \\
\hline$\left.\right|^{183}$ & $59 M$ & CABG \\
\hline $1^{117}$ & $70 M$ & CABG \\
\hline $1^{111}$ & $60 \mathrm{~F}$ & CABG \\
\hline $1^{119}$ & $61 \mathrm{M}$ & CABG \\
\hline $1^{120}$ & $71 \mathrm{M}$ & CABG \\
\hline$\left.\right|^{121}$ & $60 M$ & CABG \\
\hline $1^{115}$ & $63 \mathrm{M}$ & CABG \\
\hline $1^{115}$ & $62 M$ & CABG \\
\hline $1^{115}$ & $55 \mathrm{M}$ & CABG \\
\hline$\left.\right|^{125}$ & $56 M$ & $\begin{array}{l}\text { Mitral valve } \\
\text { replacement }\end{array}$ \\
\hline $\mathrm{I}^{126}$ & 68; gender NA & $\begin{array}{l}\text { Aortic valve } \\
\text { replacement }\end{array}$ \\
\hline$\left.\right|^{127}$ & $73 \mathrm{M}$ & $\begin{array}{l}\text { Abdominal aortic } \\
\text { aneurysm repair }\end{array}$ \\
\hline $1^{129}$ & $65 \mathrm{~F}$ & Knee arthroplasty \\
\hline $1^{130}$ & $61 M$ & Knee arthroplasty \\
\hline$\left.\right|^{114}$ & $72 M$ & Hip arthroplasty \\
\hline$\left.\right|^{130}$ & $76 M$ & Hip arthroplasty \\
\hline $1^{131}$ & $45 \mathrm{M}$ & Spine surgery \\
\hline$\left.\right|^{128}$ & $50 \mathrm{~F}$ & Liposuction \\
\hline$\left.\right|^{114}$ & $52 \mathrm{M}$ & Orchiectomy \\
\hline $1^{132}$ & $47 M$ & Cholecystectomy \\
\hline $1^{110}$ & $67 M$ & $\begin{array}{l}\text { Endoscopic sinus } \\
\text { surgery }\end{array}$ \\
\hline
\end{tabular}

Abbreviations: NA, information not available; $M$, male; F, female; $C A B G$, cardiopulmonary bypass grafting. orchiectomy; ${ }^{114}$ and endoscopic sinus surgery. ${ }^{110}$ Patients ranged in age from $45^{131}$ to 79 years. ${ }^{123}$

\section{Cortical blindness Diagnosis}

Cortical blindness results from the destruction or denervation of the primary visual cortex. The parieto-occipital region of the brain is a watershed zone for the middle cerebral and posterior cerebral arteries, and may undergo infarction during periods of systemic hypotension. Stroke after general surgery is very rare, although the risk appears to be increased after cardiac or vascular surgery, especially CABG. The majority of postoperative strokes are ischemic or embolic in nature. ${ }^{133}$ Bilateral lesions cause a syndrome with signs and symptoms ranging from bilateral lower visual field defects, difficulty in the visual judgment of size, distance, and movement, disrupted smooth oculomotor pursuit, restricted visual attention, and optic ataxia. ${ }^{134}$ Patients have reduced vision with normal pupil reaction, intact corneal reflexes, and normal eye movement. Bilateral complete vision loss is rare, and is usually seen in association with other neurologic symptoms associated with vertebrobasilar strokes, such as ataxia, hemisyndrome, diplopia, and nausea. ${ }^{135}$ Patient evaluation should include a detailed neurologic examination and brain computed tomography (CT) or MRI to evaluate the lesion. ${ }^{133}$

\section{Pathogenesis, risk factors, and outcomes}

Postoperative cortical blindness results from embolism, generalized cerebral underperfusion, or both. ${ }^{133}$ Symptoms may be immediate, identified in the first postoperative day, or delayed, presenting from the second postoperative day onward. ${ }^{136}$ Early embolism leading to stroke in CABG is thought to result from manipulations of the heart and aorta, or from the release of particulate matter from the bypass pump. Delayed embolism is generally due to postoperative atrial fibrillation, myocardial infarction, and hypercoagulability due to surgical trauma and associated tissue injury. Most strokes due to hypoperfusion are diagnosed in the first postoperative day; delayed hypoperfusion strokes are usually due to postoperative dehydration or blood loss. ${ }^{133}$ It has been suggested that repeated short episodes of extreme hypotension are more likely to result in cortical blindness than a longer episode of moderate hypotension. ${ }^{137}$ Risk factors for developing postoperative stroke include: hypertension, diabetes mellitus, renal insufficiency, smoking history, COPD, peripheral vascular disease, cardiac disease, and/or systolic dysfunction. Patients with symptomatic carotid stenosis may be at increased risk of postoperative stroke, including cortical 
blindness, and may benefit from carotid revascularization prior to undergoing surgery. ${ }^{133}$

The prognosis of postoperative parieto-occipital stroke ranges from total permanent blindness to brief periods of transient ischemic attacks with full recovery of visual acuity. ${ }^{136}$ Cortical blindness is generally seen as permanent after the window for spontaneous recovery has passed, which is thought to be several months post-lesion. ${ }^{138}$

\section{Cortical blindness literature review}

We are aware of 245 cases of postoperative cortical blindness in the literature (Table 4). Most cases were seen following CABG. ${ }^{3,51,134,136,137,139,140}$ Cases were also seen following: aortic arch repair; ${ }^{139}$ aortic valve replacement; ${ }^{134}$ radical neck dissection; ${ }^{1}$ total hip arthroplasty: ${ }^{1,3,141}$ knee replacement; ${ }^{3}$

Table 4 Cortical blindness case reports

\begin{tabular}{|c|c|c|}
\hline $\begin{array}{l}\text { Number } \\
\text { of patients, } \\
\text { reference }\end{array}$ & $\begin{array}{l}\text { Age, } \\
\text { gender }\end{array}$ & Surgery performed \\
\hline $215^{48}$ & NA & $\begin{array}{l}\text { Spinal, orthopedic, cardiac, } \\
\text { and general surgery }\end{array}$ \\
\hline $1^{136}$ & $51 \mathrm{M}$ & CABG \\
\hline $1^{136}$ & $63 \mathrm{~F}$ & CABG \\
\hline $1^{136}$ & $50 M$ & CABG \\
\hline $1^{136}$ & $58 \mathrm{~F}$ & CABG \\
\hline $1^{136}$ & $46 M$ & CABG \\
\hline$I^{136}$ & $62 M$ & CABG \\
\hline $1^{136}$ & $53 \mathrm{M}$ & CABG \\
\hline $1^{136}$ & $49 M$ & CABG \\
\hline $1^{136}$ & $46 M$ & CABG \\
\hline $1^{136}$ & $58 \mathrm{M}$ & CABG \\
\hline $1^{137}$ & $35 \mathrm{M}$ & CABG \\
\hline $1^{137}$ & $51 \mathrm{M}$ & CABG \\
\hline$\left.\right|^{137}$ & $66 M$ & CABG \\
\hline $1^{137}$ & $48 M$ & CABG \\
\hline $1^{51}$ & $67 M$ & CABG \\
\hline $1^{51}$ & $67 \mathrm{M}$ & CABG \\
\hline $1^{140}$ & $56 \mathrm{M}$ & CABG \\
\hline$I^{134}$ & $35 \mathrm{M}$ & CABG \\
\hline $1^{134}$ & $52 M$ & CABG \\
\hline$I^{134}$ & $51 \mathrm{~F}$ & Aortic valve replacement \\
\hline $1^{139}$ & $31 \mathrm{M}$ & Aortic arch repair \\
\hline${ }^{a} 3^{36}$ & $\begin{array}{l}\text { I2-68; } \\
\text { gender NA }\end{array}$ & Spine surgery \\
\hline $1^{135}$ & $66 \mathrm{~F}$ & Spine surgery \\
\hline $1^{9}$ & $58 \mathrm{~F}$ & Spine surgery \\
\hline $1^{9}$ & $57 \mathrm{M}$ & Spine surgery \\
\hline$\left.\right|^{141}$ & $51 \mathrm{~F}$ & Hip arthroplasty \\
\hline$I^{\prime}$ & $73 \mathrm{~F}$ & Hip arthroplasty \\
\hline $\mathrm{I}^{\prime}$ & $77 \mathrm{M}$ & Neck dissection \\
\hline
\end{tabular}

Notes: aStudy contains information compiled from data submitted to registry, or compiled case reports, which may be redundant with that seen in other papers listed.

Abbreviations: NA, information not available; $M$, male; F, female; CABG, cardiopulmonary bypass grafting. cholecystectomy; ${ }^{3}$ appendectomy; ${ }^{3}$ colorectal resection; ${ }^{3}$ and spine surgery. ${ }^{3,9,36,135}$ Patients ranged in age from $31^{139}$ to 77 years. $^{1}$

\section{Neuro-ophthalmologic causes of vision loss after ocular surgery}

Neuro-ophthalmologic complications of ocular surgery include optic neuropathies such as AION, PION, and direct trauma to the optic nerve. Vision loss following ocular surgery due to CRAO, central retinal vein occlusion (CRVO), globe rupture, retinal detachment, and retrobulbar hemorrhage occur much more commonly and will not be discussed here. The majority of large-scale incidence data for vision loss is for orbital surgery, such as lateral decompressions and cavernous hemangioma removal, rather than for ocular surgery. The incidence of POVL appears to be much lower following ocular surgery when compared to orbital surgery. In a review of 67 lateral decompression surgeries, there were seven cases of unilateral POVL (2.99\%). ${ }^{142}$ Another review found 14 cases of POVL out of 2500 orbital surgeries $(0.56 \%)$, with all 14 occurring during 1150 orbital exploration surgeries (1.22\%). ${ }^{143}$ We are not aware of any large-scale reviews providing incidence measurements for POVL following ocular surgery.

\section{Traumatic optic neuropathy Diagnosis}

Periocular injection of anesthetic can result in direct trauma to the optic nerve, especially following retrobulbar injection, though they may also occur following peribulbar or sub-Tenon's injection. Other potential complications of retrobulbar injection include retrobulbar hemorrhage, central retinal artery or vein occlusion, brain stem anesthesia, ${ }^{144}$ or globe rupture with retinal detachment, ${ }^{145}$ and will not be discussed here.

Postoperative vision loss from trauma to the optic nerve occurs immediately. An orbital MRI is important for the proper diagnosis in patients presenting with symptoms of optic neuropathy in the first 24 hours following periocular injection. Orbital MRI may show focal enlargement of the optic nerve or enhancement of the optic nerve sheath with gadolinium. The bright ring of signal from cerebrospinal fluid may be absent around the damaged optic nerve on $\mathrm{T}_{2}$-weighted images. ${ }^{146}$ The MRI may also demonstrate normal results.

\section{Prevention, treatment, and outcomes}

The sub-Tenon's "blind" insertion technique has been shown to be safer than either retrobulbar or peribulbar injections, 
while still providing equally effective anesthesia and akinesia. This is believed to be due to the cannula's relatively short length and blunt tip, which limits damage to the optic nerve. ${ }^{147-149}$ There have been rare reports of optic nerve trauma following sub-Tenon's injection; therefore changing the mode of injection alone is not enough to prevent the occurrence of optic nerve trauma. ${ }^{150}$ Corticosteroid administration may relieve optic nerve swelling and restore visual acuity, and a trial of corticosteroids should be considered in patients presenting with evidence of direct needle trauma postoperatively. ${ }^{146}$

\section{Direct optic nerve trauma literature review}

We are aware of five reported cases of optic nerve trauma following ocular surgery. Most cases were following retrobulbar injection, ${ }^{151-154}$ although cases were also seen following peribulbar ${ }^{155}$ and sub-Tenon's injection. ${ }^{150}$ One patient showed partial recovery of visual acuity after receiving corticosteroid therapy. ${ }^{153}$ Three other patients showed no improvement despite receiving corticosteroids ${ }^{152,154}$ (follow-up information is unknown for one patient). ${ }^{150}$

\section{Ischemic optic neuropathies}

While rare, there have been a number of documented cases of AION or PION developing after ocular surgery with the use of periocular or general anesthesia. A retrospective review of 5787 cataract extractions found two cases of AION within six weeks of surgery. However, one patient had a history of spontaneous AION in the contralateral eye 21 months prior. ${ }^{156}$

\section{Pathogenesis, risk factors, and outcomes}

The pathogenesis of AION after ocular surgery remains controversial. It has been postulated that elevated intraocular pressure, in eyes with vulnerable optic nerve circulation, may promote ischemia of the optic nerve head following cataract extraction. ${ }^{157}$ However, there have been many cases of ION development in eyes with normal IOP. ${ }^{158-160}$ Elevated IOP has also been postulated to play a role in the development of AION following laser in-situ keratomileusis (LASIK). ${ }^{162}$ In order to create a lamellar flap during LASIK, the IOP must be raised to $>65 \mathrm{mmHg}$ by a suction ring on the anterior segment of the eye. ${ }^{162}$ However, AION has also been documented following the use of a femtosecond laser flap with low suction ring, a technique that maintains the IOP at or below $30-40 \mathrm{mmHg},{ }^{163}$ and in another patient whose IOP was maintained at $25 \mathrm{mmHg}$ through the administration of glaucoma medication. ${ }^{164}$ It is worth noting that both of these patients had small cup-to-disc ratios in the fellow eye, a proposed risk factor for AION development. ${ }^{165}$ Some have suggested that AION occurring after ocular surgery is coincidental in nature. However, one study compared the likelihood of developing AION 0-6 months or 6-12 months after cataract extraction found that all 18 cases of AION were within the first 6 postoperative months, which differed significantly $(P<0.001)$ from the uniform distribution expected if there were no temporal relationship to surgery. ${ }^{166}$ Approximately $50 \%$ of patients who develop AION following cataract extraction who undergo contralateral extraction at a later date developed AION in the fellow eye, compared to $19 \%$ in a control group who did not have cataract surgery. ${ }^{167}$ This suggests that some patients may be predisposed to developing AION after ocular surgery.

No treatment has been proven effective in the management of postoperative ischemic optic neuropathies. Many patients report spontaneous improvement in visual acuity, although none enjoy complete improvement. ${ }^{146}$ Improvements in microkeratome design with shorter suction times and faster cutting have caused a decrease in the incidence of optic nerve damage after LASIK. ${ }^{168}$ Contralateral cataract extraction should be approached with caution in patients who develop ION postoperatively, as these patients are at increased risk of developing ION in the fellow eye.

\section{Anterior ischemic optic neuropathy literature review}

We are aware of 43 cases in the literature of AION occurring after ocular surgery. The most common surgery was cataract extraction, ${ }^{156,157,169-171}$ followed by LASIK. ${ }^{164,172,173}$ Cases were also seen following vitrectomy, ${ }^{174}$ scleral buckle, ${ }^{175}$ pneumoplethysmography, ${ }^{176}$ and strabismus surgery. ${ }^{177}$ Patients ranged in age from $26^{177}$ to 94 years old. ${ }^{174}$

\section{Posterior ischemic optic neuropathy literature review}

We are aware of five cases in the literature of PION occurring after ocular surgery. The most common surgery was LASIK. ${ }^{162,164}$ There was also one case following blepharoplasty $^{178}$ and one case following cataract extraction. ${ }^{179}$ Patients ranged in age from $29^{164}$ to 78 years. ${ }^{179}$

\section{Conclusions}

Potential neuro-ophthalmologic complications following nonocular surgery include anterior ischemic optic neuropathy, posterior ischemic optic neuropathy, central retinal artery 
occlusion, pituitary apoplexy, and cortical blindness. Reports have attempted to connect anemia, hypotension, blood loss, and other hemodynamic variables to the pathophysiology of AION and PION. These variables occur in nearly all cases of cardiac and spine surgery and yet, AION and PION occur rarely. There may be an individual predisposition of certain patients, or multiple factors that lead to a "perfect storm" of events resulting in AION or PION. Meanwhile CRAO almost always occurs following spinal surgery in the prone position, where ocular compression by the headrest prevents ocular perfusion.

Cortical blindness occurs most frequently after cardiac or vascular surgery, and may be due to embolism or general cerebral underperfusion. Pituitary apoplexy is seen most often following cardiac surgery in patients with pre-existing pituitary adenomas. Causes likely include fluctuations in blood pressure, hypotension, blood dilution with crystalloid, anticoagulation, excessive steroid secretion, and transient increases in intracranial pressure. While there are currently no established treatments available for AION, PION, CRAO, or cortical blindness, patients with pituitary apoplexy may benefit from urgent transsphenoidal decompression surgery and corticosteroid administration.

Potential neuro-ophthalmologic complications following ocular surgery include traumatic optic neuropathy, anterior ischemic optic neuropathy, and posterior ischemic optic neuropathy. Traumatic optic neuropathy is seen most frequently following retrobulbar injection, although it may also occur after peribulbar, or even sub-Tenon's, injection. Diagnosis can often be confirmed with orbital MRI, and visual acuity may improve with corticosteroid administration. AION is seen most often after cataract extraction or laser in-situ keratomileusis, and may be linked to elevated intraocular pressure during surgery. PION is seen most often following LASIK. There is no established treatment available for AION or PION. Contralateral cataract extraction requires careful consideration and patient counseling in patients who develop AION after unilateral extraction, because of the increased risk of developing of AION in the fellow eye.

\section{Disclosure}

The authors report no conflicts of interest in this work.

\section{References}

1. Warner ME, Warner MA, Garrity JA, MacKenzie RA, Warner DO. The frequency of perioperative vision loss. Anesth Analg. 2001;93(6): 1417-1421.

2. Roth S, Thisted RA, Erickson JP, Black S, Schreider BD. Eye injuries after nonocular surgery. A study of 60,965 anesthetics from 1988 to 1992. Anesthesiology. 1996;85(5):1020-1027.
3. Shen Y, Drum M, Roth S. The prevalence of perioperative visual loss in the United States: a 10-year study from 1996 to 2005 of spinal, orthopedic, cardiac, and general surgery. Anesth Analg. 2009;109(5): 1534-1545.

4. Nuttall GA, Garrity JA, Dearani JA, Abel MD, Schroeder DR, Mullany CJ. Risk factors for ischemic optic neuropathy after cardiopulmonary bypass: a matched case/control study. Anesth Analg. 2001;93(6):1410-1416.

5. Sweeney PJ, Breuer AC, Selhorst JB, et al. Ischemic optic neuropathy: a complication of cardiopulmonary bypass surgery. Neurology. 1982;32(5):560-562.

6. Kalyani SD, Miller NR, Dong LM, Baumgartner WA, Alejo DE, Gilbert TB. Incidence of and risk factors for perioperative optic neuropathy after cardiac surgery. Ann Thorac Surg. 2004;78(1): 34-37.

7. Roth S, Barach P. Postoperative visual loss: still no answers - yet. Anesthesiology. 2001;95(3):575-577.

8. Patil CG, Lad EM, Lad SP, Ho C, Boakye M. Visual loss after spine surgery: a population-based study. Spine (Phila Pa 1976). 2008;33(13):1491-1496.

9. Stevens WR, Glazer PA, Kelley SD, Lietman TM, Bradford DS. Ophthalmic complications after spinal surgery. Spine (Phila Pa 1976). 1997;22(12):1319-1324.

10. Lee LA, Roth S, Posner KL, et al. The American Society of Anesthesiologists Postoperative Visual Loss Registry: analysis of 93 spine surgery cases with postoperative visual loss. Anesthesiology. 2006;105(4):652-659.

11. Williams EL, Hart WM Jr, Tempelhoff R. Postoperative ischemic optic neuropathy. Anesth Analg. 1995;80(5):1018-1029.

12. Holy SE, Tsai JH, McAllister RK, Smith KH. Perioperative ischemic optic neuropathy: a case control analysis of 126,666 surgical procedures at a single institution. Anesthesiology. 2009;110(2):246-253.

13. Buono LM, Foroozan R. Perioperative posterior ischemic optic neuropathy: review of the literature. Surv Ophthalmol. 2005;50(1):15-26.

14. Chang SH, Miller NR. The incidence of vision loss due to perioperative ischemic optic neuropathy associated with spine surgery: the Johns Hopkins Hospital Experience. Spine (Phila Pa 1976). 2005;30(11):1299-1302.

15. Shapira OM, Kimmel WA, Lindsey PS, Shahian DM. Anterior ischemic optic neuropathy after open heart operations. Ann Thorac Surg. 1996;61(2):660-666.

16. Lessell S. Nonarteritic anterior ischemic optic neuropathy: enigma variations. Arch Ophthalmol. 1999;117(3):386-388.

17. Miller NR. Anterior ischemic optic neuropathy: diagnosis and management. Bull NYAcad Med. 1980;56(7):643-654.

18. Hayreh SS. Anterior ischemic optic neuropathy. Clin Neurosci. 1997;4(5):251-263.

19. Alpert JN, Pena Y, Leachman DR. Anterior ischemic optic neuropathy after coronary bypass surgery. Tex Med. 1987;83(8):45-47.

20. Sadda SR, Nee M, Miller NR, Biousse V, Newman NJ, Kouzis A. Clinical spectrum of posterior ischemic optic neuropathy. $\mathrm{Am} \mathrm{J}$ Ophthalmol. 2001;132(5):743-750.

21. Vaphiades MS. Optic nerve enhancement in hypotensive ischemic optic neuropathy. J Neuroophthalmol. 2004;24(3):235-236.

22. Purvin V, Kuzma B. Intraorbital optic nerve signal hyperintensity on magnetic resonance imaging sequences in perioperative hypotensive ischemic optic neuropathy. J Neuroophthalmol. 2005;25(3):202-204.

23. Johnson MW, Kincaid MC, Trobe JD. Bilateral retrobulbar optic nerve infarctions after blood loss and hypotension. A clinicopathologic case study. Ophthalmology. 1987;94(12):1577-1584.

24. Hayreh SS. Anterior ischemic optic neuropathy. VIII. Clinical features and pathogenesis of post-hemorrhagic amaurosis. Ophthalmology. 1987;94(11):1488-1502.

25. Rath EZ, FalickY, Rumelt S. Posterior ischemic optic neuropathy following breast augmentation and abdominal liposuction. Can J Ophthalmol. 2009;44(3):346-347.

26. Metwalli AR, Davis RG, Donovan JF. Visual impairment after laparoscopic donor nephrectomy. J Endourol. 2004;18(9):888-890. 
27. Reuler JB. Hypothermia: pathophysiology, clinical settings, and management. Ann Intern Med. 1978;89(4):519-527.

28. Chenoweth DE, Cooper SW, Hugli TE, Stewart RW, Blackstone EH, Kirklin JW. Complement activation during cardiopulmonary bypass evidence for generation of $\mathrm{C} 3 \mathrm{a}$ and $\mathrm{C} 5 \mathrm{a}$ anaphylatoxins. $N$ Engl J Med. 1981;304(9):497-503.

29. Katz B. Anterior ischemic optic neuropathy and intraocular pressure. Arch Ophthalmol. 1992;110(5):596-597.

30. Tomsak RL, Remler BF. Anterior ischemic optic neuropathy and increased intraocular pressure. $J$ Clin Neuroophthalmol. 1989;9(2):116-118.

31. Larkin DF, Connolly P, Magner JB, Wood AE, Eustace P. Intraocular pressure during cardiopulmonary bypass. $\mathrm{Br} J$ Ophthalmol. 1987;71(3):177-180.

32. Deutch D, Lewis RA. Intraocular pressure after cardiopulmonary bypass surgery. Am J Ophthalmol. 1989;107(1):18-22.

33. Hayreh SS. Factors influencing blood flow in the optic nerve head J Glaucoma. 1997;6(6):412-425.

34. Haefliger IO, Meyer P, Flammer J, Luscher TF. The vascular endothelium as a regulator of the ocular circulation: a new concept in ophthalmology? Surv Ophthalmol. 1994;39(2):123-132.

35. Isayama $\mathrm{Y}$, Takahashi $\mathrm{T}$, Inoue $\mathrm{M}$, Jimura T. Posterior ischemic optic neuropathy. III. Clinical diagnosis. Ophthalmologica. 1983;187(3): 141-147.

36. Myers MA, Hamilton SR, Bogosian AJ, Smith CH, Wagner TA. Visual loss as a complication of spine surgery. A review of 37 cases. Spine (Phila Pa 1976). 1997;22(12):1325-1329.

37. Dunker S, Hsu HY, Sebag J, Sadun AA. Perioperative risk factors for posterior ischemic optic neuropathy. J Am Coll Surg. 2002;194(6):705-710.

38. Hunt K, Bajekal R, Calder I, Meacher R, Eliahoo J, Acheson JF Changes in intraocular pressure in anesthetized prone patients J Neurosurg Anesthesiol. 2004;16(4):287-290.

39. Lam AK, Douthwaite WA. Does the change of anterior chamber depth or/and episcleral venous pressure cause intraocular pressure change in postural variation? Optom Vis Sci. 1997;74(8):664-667.

40. Wolfe SW, Lospinuso MF, Burke SW. Unilateral blindness as a complication of patient positioning for spinal surgery. A case report. Spine (Phila Pa 1976). 1992;17(5):600-605.

41. Cheng MA, Sigurdson W, Tempelhoff R, Lauryssen C. Visual loss after spine surgery: a survey. Neurosurgery. 2000;46(3):625-631.

42. Heitz JW, Audu PB. Asymmetric postoperative visual loss after spine surgery in the lateral decubitus position. $\mathrm{Br} J$ Anaesth 2008;101(3):380-382.

43. Marks SC, Jaques DA, Hirata RM, Saunders JR Jr. Blindness following bilateral radical neck dissection. Head Neck. 1990;12(4):342-345.

44. Kawasaki A, Purvin V. Recovery of postoperative visual loss following treatment of severe anaemia. Clin Experiment Ophthalmol. 2006;34(5):497-499.

45. Connolly SE, Gordon KB, Horton JC. Salvage of vision after hypotension-induced ischemic optic neuropathy. Am J Ophthalmol. 1994;117(2):235-242.

46. Lee LA, Lam AM. Unilateral blindness after prone lumbar spine surgery. Anesthesiology. 2001;95(3):793-795.

47. Breuer AC, Furlan AJ, Hanson MR, et al. Central nervous system complications of coronary artery bypass graft surgery: prospective analysis of 421 patients. Stroke. 1983;14(5):682-687.

48. Shen Y, Drum M, Roth S. The prevalence of perioperative visual loss in the United States: a 10-year study from 1996 to 2005 of spinal, orthopedic, cardiac, and general surgery. Anesth Analg. 2009;109(5):1534-1545

49. Robinson RJ, Cecere R, Chiu RC. Binocular blindness following dynamic cardiomyoplasty. J Card Surg. 1996;11(1):75-78.

50. Larkin DF, Wood AE, Neligan M, Eustace P. Ischaemic optic neuropathy complicating cardiopulmonary bypass. $\mathrm{Br} J$ Ophthalmol. 1987;71(5):344-347.

51. Shahian DM, Speert PK. Symptomatic visual deficits after open heart operations. Ann Thorac Surg. 1989;48(2):275-279.
52. Tomsak RL. Ischemic optic neuropathy associated with retinal embolism. Am J Ophthalmol. 1985;99(5):590-592.

53. Moster ML. Visual loss after coronary artery bypass surgery. Surv Ophthalmol. 1998;42(5):453-457.

54. Sha'aban RI, Asfour WM. Visual loss after coronary artery bypass surgery. Saudi Med J. 2000;21(1):90-92.

55. Jaben SL, Glaser JS, Daily M. Ischemic optic neuropathy following general surgical procedures. J Clin Neuroophthalmol. 1983;3(4):239-244.

56. Busch T, Sirbu H, Aleksic I, Stamm C, Zenker D, Dalichau H. Anterior ischemic optic neuropathy: a complication after extracorporal circulation. Ann Thorac Cardiovasc Surg. 1998;4(6):354-358.

57. Tidow-Kebritchi S, Jay WM. Anterior ischemic optic neuropathy following off-pump cardiac bypass surgery. Semin Ophthalmol. 2003;18(4):166-168.

58. Katzman SS, Moschonas CG, Dzioba RB. Amaurosis secondary to massive blood loss after lumbar spine surgery. Spine (Phila Pa 1976). 1994;19(4):468-469.

59. West J, Askin G, Clarke M, Vernon SA. Loss of vision in one eye following scoliosis surgery. Br J Ophthalmol. 1990;74(4): 243-244.

60. Kim JW, Hills WL, Rizzo JF, Egan RA, Lessell S. Ischemic optic neuropathy following spine surgery in a 16-year-old patient and a tenyear-old patient. J Neuroophthalmol. 2006;26(1):30-33.

61. Dilger JA, Tetzlaff JE, Bell GR, Kosmorsky GS, Agnor RC, O’Hara JF Jr. Ischaemic optic neuropathy after spinal fusion. Can J Anaesth. 1998;45(1):63-66.

62. Katz DM, Trobe JD, Cornblath WT, Kline LB. Ischemic optic neuropathy after lumbar spine surgery. Arch Ophthalmol. 1994; 112(7):925-931.

63. Tice DA. Ischemic optic neuropathy and cardiac surgery. Ann Thorac Surg. 1987;44(6):677.

64. Fournier JH, Velis E. Visual loss after shoulder surgery under general anesthesia diagnosed as caused by ocular compression with electroretinography testing: case report and review of the literature. Int Surg. 2004;89(4):209-211.

65. Gilbert ME, Savino PJ, Sergott RC. Anterior ischaemic optic neuropathy after rotator cuff surgery. Br J Ophthalmol. 2006;90(2):248-249.

66. Aydin O, Memisoglu I, Ozturk M, Altintas O. Anterior ischemic optic neuropathy after unilateral radical neck dissection: case report and review. Auris Nasus Larynx. 2008;35(2):308-312.

67. Gotte K, Riedel F, Knorz MC, Hormann K. Delayed anterior ischemic optic neuropathy after neck dissection. Arch Otolaryngol Head Neck Surg. 2000;126(2):220-223.

68. Chutkow JG, Sharbrough FW, Riley FC Jr. Blindness following simultaneous bilateral neck dissection. Mayo Clin Proc. 1973;48(10):713-717.

69. Wilson JF, Freeman SB, Breene DP. Anterior ischemic optic neuropathy causing blindness in the head and neck surgery patient. Arch Otolaryngol Head Neck Surg. 1991;117(11):1304-1306.

70. Strome SE, Hill JS, Burnstine MA, Beck J, Chepeha DB, Esclamado RM. Anterior ischemic optic neuropathy following neck dissection. Head Neck. 1997;19(2):148-152.

71. Brown RH, Schauble JF, Miller NR. Anemia and hypotension as contributors to perioperative loss of vision. Anesthesiology. 1994;80(1):222-226.

72. Sadaba LM, Garcia-Layana A, Maldonado MJ, Berian JM. Bilateral ischemic optic neuropathy after transurethral prostatic resection: a case report. BMC Ophthalmol. 2006;6:32.

73. Williams GC, Lee AG, Adler HL, et al. Bilateral anterior ischemic optic neuropathy and branch retinal artery occlusion after radical prostatectomy. J Urol. 1999;162(4):1384-1385.

74. Kaeser PF, Borruat FX. Visual Loss After Orthopedic Procedures. J Arthroplasty. 2010 Feb 8. [Epub ahead of print].

75. Minagar A, Schatz NJ, Glaser JS. Liposuction and ischemic optic neuropathy. Case report and review of literature. J Neurol Sci. 2000;181(1-2):132-136.

76. Foroozan R, Varon J. Bilateral anterior ischemic optic neuropathy after liposuction. J Neuroophthalmol. 2004;24(3):211-213. 
77. Roth S, Nunez R, Schreider BD. Unexplained visual loss after lumbar spinal fusion. J Neurosurg Anesthesiol. 1997;9(4):346-348.

78. Murphy MA. Bilateral posterior ischemic optic neuropathy after lumbar spine surgery. Ophthalmology. 2003;110(7):1454-1457.

79. Abraham M, Sakhuja N, Sinha S, Rastogi S. Unilateral visual loss after cervical spine surgery. J Neurosurg Anesthesiol. 2003; 15(4):319-322.

80. Gaillard MC, Zambaz BD, Borruat FX. Posterior ischemic optic neuropathy: case report of a rare complication after general surgery. Klin Monbl Augenheilkd. 2004;221(5):421-423.

81. Lee AG. Ischemic optic neuropathy following lumbar spine surgery. Case report. J Neurosurg. 1995;83(2):348-349.

82. Alexandrakis G, Lam BL. Bilateral posterior ischemic optic neuropathy after spinal surgery. Am J Ophthalmol. 1999;127(3):354-355.

83. Kamming D, Clarke S. Postoperative visual loss following prone spinal surgery. Br J Anaesth. 2005;95(2):257-260.

84. Pazos GA, Leonard DW, Blice J, Thompson DH. Blindness after bilateral neck dissection: case report and review. Am J Otolaryngol. 1999;20(5):340-345.

85. Schobel GA, Schmidbauer M, Millesi W, Undt G. Posterior ischemic optic neuropathy following bilateral radical neck dissection. Int J Oral Maxillofac Surg. 1995;24(4):283-287.

86. Balm AJ, Brown DH, De Vries WA, Snow GB. Blindness: a potential complication of bilateral neck dissection. $J$ Laryngol Otol. 1990;104(2):154-156.

87. Kirkali P, Kansu T. A case of unilateral posterior ischemic optic neuropathy after radical neck dissection. Ann Ophthalmol. 1990;22(8):297-298.

88. Rizzo JF 3rd, Lessell S. Posterior ischemic optic neuropathy during general surgery. Am J Ophthalmol. 1987;103(6):808-811.

89. Bhatti MT, Enneking FK. Visual loss and ophthalmoplegia after shoulder surgery. Anesth Analg. 2003;96(3):899-902, table of contents.

90. Remigio D, Wertenbaker C. Postoperative bilateral vision loss. Surv Ophthalmol. 2000;44(5):426-432.

91. Plate S, Asboe S. Blindness as a complication of rhinosurgery. J Laryngol Otol. 1981;95(3):317-322.

92. Noble MJ, Alvarez EV. Combined occlusion of the central retinal artery and central retinal vein following blunt ocular trauma: a case report. Br J Ophthalmol. 1987;71(11):834-836.

93. Kollarits CR, Lubow M, Hissong SL. Retinal strokes. I. Incidence of carotid atheromata. JAMA. 1972;222(10):1273-1275.

94. Brown GC, Magargal LE, Shields JA, Goldberg RE, Walsh PN. Retinal arterial obstruction in children and young adults. Ophthalmology. 1981;88(1):18-25.

95. Grossman W, Ward WT. Central retinal artery occlusion after scoliosis surgery with a horseshoe headrest. Case report and literature review. Spine (Phila Pa 1976). 1993;18(9):1226-1228.

96. Hayreh SS, Kolder HE, Weingeist TA. Central retinal artery occlusion and retinal tolerance time. Ophthalmology. 1980;87(1):75-78.

97. Nakra D, Bala I, Pratap M. Unilateral postoperative visual loss due to central retinal artery occlusion following cervical spine surgery in prone position. Paediatr Anaesth. 2007;17(8):805-808.

98. Hoski JJ, Eismont FJ, Green BA. Blindness as a complication of intraoperative positioning. A case report. J Bone Joint Surg Am. 1993;75(8):1231-1232.

99. Bekar A, Tureyen K, Aksoy K. Unilateral blindness due to patient positioning during cervical syringomyelia surgery: unilateral blindness after prone position. J Neurosurg Anesthesiol. 1996;8(3):227-229.

100. Mertens E, Smets RM, Sys J, Michielsen J, Verstreken J, Tassignon MJ. Central retinal artery occlusion after back surgery: a case report. Bull Soc Belge Ophtalmol. 1995;255:127-131.

101. Sys J, Michielsen J, Mertens E, Verstreken J, Tassignon MJ. Central retinal artery occlusion after spinal surgery. Eur Spine J. 1996;5(1):74-75.

102. Bradish CF, Flowers M. Central retinal artery occlusion in association with osteogenesis imperfecta. Spine (Phila Pa 1976). 1987;12(2):193-194.
103. Stambough JL, Cheeks ML. Central retinal artery occlusion: a complication of the knee-chest position. J Spinal Disord. 1992;5(3): 363-365.

104. Manfredini M, Ferrante R, Gildone A, Massari L. Unilateral blindness as a complication of intraoperative positioning for cervical spinal surgery. J Spinal Disord. 2000;13(3):271-272.

105. Pandey V, Rao PS, Rao SK, Acharya KK. Monocular blindness due to central retinal artery occlusion in bipolar hemireplacement arthroplasty of the hip. Singapore Med J. 2008;49(4):e96-e97.

106. Sibal L, Ball SG, Connolly V, et al. Pituitary apoplexy: a review of clinical presentation, management and outcome in 45 cases. Pituitary. 2004;7(3):157-163.

107. Randeva HS, Schoebel J, Byrne J, Esiri M, Adams CB, Wass JA. Classical pituitary apoplexy: clinical features, management and outcome. Clin Endocrinol (Oxf). 1999;51(2):181-188.

108. Kovacs K, Yao J. Pituitary necrosis following major heart surgery. Z Kardiol. 1975;64(1):52-57.

109. Biousse V, Newman NJ, Oyesiku NM. Precipitating factors in pituitary apoplexy. J Neurol Neurosurg Psychiatry. 2001;71(4):542-545.

110. Fyrmpas G, Constantinidis J, Foroglou N, Selviaridis P. Pituitary apoplexy following endoscopic sinus surgery. J Laryngol Otol. 2009; Nov 25:1-3. [Epub ahead of print].

111. Shapiro LM. Pituitary apoplexy following coronary artery bypass surgery. J Surg Oncol. 1990;44(1):66-68.

112. Bills DC, Meyer FB, Laws ER Jr, et al. A retrospective analysis of pituitary apoplexy. Neurosurgery. 1993;33(4):602-609.

113. Ayuk J, McGregor EJ, Mitchell RD, Gittoes NJ. Acute management of pituitary apoplexy - surgery or conservative management? Clin Endocrinol (Oxf). 2004;61(6):747-752.

114. Semple PL, Jane JA Jr, Laws ER Jr. Clinical relevance of precipitating factors in pituitary apoplexy. Neurosurgery. 2007;61(5):956-962.

115. Cooper DM, Bazaral MG, Furlan AJ, et al. Pituitary apoplexy: a complication of cardiac surgery. Ann Thorac Surg. 1986;41(5): 547-550.

116. Slavin ML, Budabin M. Pituitary apoplexy associated with cardiac surgery. Am J Ophthalmol. 1984;98(3):291-296.

117. Telesca M, Santini F, Mazzucco A. Adenoma related pituitary apoplexy disclosed by ptosis after routine cardiac surgery: occasional reappearance of a dismal complication. Intensive Care Med. 2009;35(1):185-186.

118. Levy E, Korach A, Merin G, Feinsod M, Glenville B. Pituitary apoplexy and CABG: should we change our strategy? Ann Thorac Surg. 2007;84(4):1388-1390.

119. Absalom M, Rogers KH, Moulton RJ, Mazer CD. Pituitary apoplexy after coronary artery surgery. Anesth Analg. 1993;76(3): 648-649.

120. Mukhida K, Kolyvas G. Pituitary apoplexy following cardiac surgery. Can J Neurol Sci. 2007;34(3):390-393.

121. Loubani M, Galinanes M. Pituitary gland macroadenoma: a cause of transient blindness after cardiac surgery. Ann Thorac Surg. 2001;72(3):929-931.

122. Savage EB, Gugino L, Starr PA, Black PM, Cohn LH, Aranki SF. Pituitary apoplexy following cardiopulmonary bypass: considerations for a staged cardiac and neurosurgical procedure. Eur J Cardiothorac Surg. 1994;8(6):333-336.

123. Thurtell MJ, Besser M, Halmagyi GM. Pituitary apoplexy causing isolated blindness after cardiac bypass surgery. Arch Ophthalmol. 2008;126(4):576-578.

124. Pliam MB, Cohen M, Cheng L, Spaenle M, Bronstein MH, Atkin TW. Pituitary adenomas complicating cardiac surgery: summary and review of 11 cases. J Card Surg. 1995;10(2):125-132.

125. Meek EN, Butterworth J, Kon ND, Zvara DA, Ash GE Jr, Martin TJ. Pituitary apoplexy following mitral valve repair. Anesthesiology. 1998;89(6):1580-1582.

126. Peck V, Lieberman A, Pinto R, Culliford A. Pituitary apoplexy following open-heart surgery. NY State J Med. 1980;80(4):641-643. 
127. Liberale G, Bruninx G, Vanderkelen B, Dubois E, Vandueren E, Verhelst G. Pituitary apoplexy after aortic abdominal aneurysm surgery: a case report. Acta Chir Belg. 2006;106(1):77-80.

128. Cohen A, Kishore K, Wolansky L, Frohman L. Pituitary apoplexy occurring during large volume liposuction surgery. J Neuroophthalmol. 2004;24(1):31-33.

129. Khandelwal M, Chhabra A, Krishnan S. Pituitary apoplexy following bilateral total knee arthroplasty. J Postgrad Med. 2005;51(2): $155-156$.

130. Goel V, Debnath UK, Singh J, Brydon HL. Pituitary apoplexy after joint arthroplasty. J Arthroplasty. 2009;24(5):826.e7-826.e10.

131. Liu JK, Nwagwu C, Pikus HJ, Couldwell WT. Laparoscopic anterior lumbar interbody fusion precipitating pituitary apoplexy. Acta Neurochir (Wien). 2001;143(3):303-307.

132. Yahagi N, Nishikawa A, Matsui S, Komoda Y, Sai Y, Amakata Y. Pituitary apoplexy following cholecystectomy. Anaesthesia. 1992; 47(3):234-236.

133. Selim M. Perioperative stroke. N Engl J Med. 2007;356(7): 706-713.

134. Howard R, Trend P, Russell RW. Clinical features of ischemia in cerebral arterial border zones after periods of reduced cerebral blood flow. Arch Neurol. 1987;44(9):934-940.

135. Huber JF, Grob D. Bilateral cortical blindness after lumbar spine surgery. A case report. Spine (Phila Pa 1976). 1998;23(16):1807-1809.

136. Taugher PJ. Visual loss after cardiopulmonary bypass. Am J Ophthalmol. 1976;81(3):280-288.

137. Russell RW, Bharucha N. The recognition and prevention of border zone cerebral ischaemia during cardiac surgery. $Q \mathrm{~J} \mathrm{Med}$. 1978;47(187):303-323

138. Stoerig P. Functional rehabilitation of partial cortical blindness? Restor Neurol Neurosci. 2008;26(4-5):291-303.

139. Suzuki Y, Kiyosawa M, Mochizuki M, Ishii K, Senda M. Cortical blindness following aortic arch surgery. Jpn J Ophthalmol. 2001;45(5):547-549.

140. Smith JL, Cross SA. Occipital lobe infarction after open heart surgery. J Clin Neuroophthalmol. 1983;3(1):23-30.

141. Gelinas JJ, Cherry R, MacDonald SJ. Fat embolism syndrome after cementless total hip arthroplasty. J Arthroplasty. 2000;15(6):809-813.

142. Long JC, Ellis PP. Total unilateral visual loss following orbital surgery. Am J Ophthalmol. 1971;1(1 Pt 2):218-220.

143. Rose GE. The "devil's touch"; visual loss and orbital surgery. A synopsis of the Mustarde Lecture, 2006. Orbit. 2007;26(3):147-158.

144. Horton JC, Hoyt WF, Foreman DS, Cohen JA. Confirmation by magnetic resonance imaging of optic nerve injury after retrobulbar anesthesia. Arch Ophthalmol. 1996;114(3):351-353.

145. Mameletzi E, Pournaras JA, Ambresin A, Nguyen C. Retinal embolisation with localised retinal detachment following retrobulbar anaesthesia. Klin Monbl Augenheilkd. 2008;225(5):476-478.

146. Lee MS, Rizzo JF 3rd, Lessell S. Neuro-ophthalmologic complications of cataract surgery. Semin Ophthalmol. 2002;17(3-4):149-152.

147. Mein CE, Woodcock MG. Local anesthesia for vitreoretinal surgery. Retina. 1990;10(1):47-49.

148. Hansen EA, Mein CE, Mazzoli R. Ocular anesthesia for cataract surgery: a direct sub-Tenon's approach. Ophthalmic Surg. 1990;21(10):696-699.

149. Stevens JD. A new local anesthesia technique for cataract extraction by one quadrant sub-Tenon's infiltration. Br J Ophthalmol. 1992;76(11):670-674.

150. Kim SK, Andreoli CM, Rizzo JF 3rd, Golden MA, Bradbury MJ. Optic neuropathy secondary to sub-tenon anesthetic injection in cataract surgery. Arch Ophthalmol. 2003;121(6):907-909.

151. Pautler SE, Grizzard WS, Thompson LN, Wing GL. Blindness from retrobulbar injection into the optic nerve. Ophthalmic Surg 1986;17(6):334-337.

152. Jindra LF. Blindness following retrobulbar anesthesia for astigmatic keratotomy. Ophthalmic Surg. 1989;20(6):433-435.
153. Horton JC, Hoyt WF, Foreman DS, Cohen JA. Confirmation by magnetic resonance imaging of optic nerve injury after retrobulbar anesthesia. Arch Ophthalmol. 1996;114(3):351-353.

154. Devoto MH, Kersten RC, Zalta AH, Kulwin DR. Optic nerve injury after retrobulbar anesthesia. Arch Ophthalmol. 1997;115(5):687-688.

155. Dorey SE, Gillespie IH, Barton F, MacSweeney E. Magnetic resonance image changes following optic nerve trauma from peribulbar anaesthetic. Br J Ophthalmol. 1998;82(5):586-587.

156. McCulley TJ, Lam BL, Feuer WJ. Incidence of nonarteritic anterior ischemic optic neuropathy associated with cataract extraction. Ophthalmology. 2001;108(7):1275-1278.

157. Hayreh SS. Anterior ischemic optic neuropathy. IV. Occurrence after cataract extraction. Arch Ophthalmol. 1980;98(8):1410-1416.

158. Hattenhauer MG, Leavitt JA, Hodge DO, Grill R, Gray DT. Incidence of nonarteritic anterior ischemic optic neuropathy. Am J Ophthalmol. 1997;123(1):103-107.

159. Carroll FD. Optic nerve complications of cataract extraction. Trans Am Acad Ophthalmol Otolaryngol. 1973;77(5):OP623-OP629.

160. Reese AB, Carroll FD. Optic neuritis following cataract extraction. Trans Am Acad Ophthalmol Otolaryngol. 1958;62(6):765-770.

161. Lee AG, Kohnen T, Ebner R, et al. Optic neuropathy associated with laser in situ keratomileusis. J Cataract Refract Surg. 2000;26(11):1581-1584.

162. Cameron BD, Saffra NA, Strominger MB. Laser in situ keratomileusisinduced optic neuropathy. Ophthalmology. 2001;108(4):660-665.

163. Maden A, Yilmaz S, Yurdakul NS. Nonarteritic ischemic optic neuropathy after LASIK with femtosecond laser flap creation. J Neuroophthalmol. 2008;28(3):242-243.

164. Lee AG. LASIK-induced optic neuropathy. Ophthalmology. 2002;109(5):817; author reply 817

165. Hayreh SS. Risk factors in AION. Ophthalmology. 2001;108(10): 1717-1718.

166. McCulley TJ, Lam BL, Feuer WJ. Nonarteritic anterior ischemic optic neuropathy and surgery of the anterior segment: temporal relationship analysis. Am J Ophthalmol. 2003;136(6):1171-1172.

167. Lam BL, Jabaly-Habib H, Al-Sheikh N, et al. Risk of non-arteritic anterior ischaemic optic neuropathy (NAION) after cataract extraction in the fellow eye of patients with prior unilateral NAION. $\mathrm{Br} J$ Ophthalmol. 2007;91(5):585-587.

168. Mirshahi A, Baatz H. Posterior segment complications of laser in situ keratomileusis (LASIK). Surv Ophthalmol. 2009;54(4):433-440.

169. Serrano LA, Behrens MM, Carroll FD. Postcataract extraction ischemic optic neuropathy. Arch Ophthalmol. 1982;100(7):1177-1178.

170. Michaels DD, Zugsmith GS. Optic neuropathy following cataract extraction. Ann Ophthalmol. 1973;5(3):303-306.

171. Spedick MJ, Tomsak RL. Ischemic optic neuropathy following secondary intraocular lens implantation. J Clin Neuroophthalmol. 1984;4(4):255-257.

172. Maden A, Yilmaz S, Yurdakul NS. Nonarteritic ischemic optic neuropathy after LASIK with femtosecond laser flap creation. J Neuroophthalmol. 2008;28(3):242-243.

173. Bushley DM, Parmley VC, Paglen P. Visual field defect associated with laser in situ keratomileusis. Am J Ophthalmol. 2000;129(5):668-671.

174. Taban M, Lewis H, Lee MS. Nonarteritic anterior ischemic optic neuropathy and 'visual field defects' following vitrectomy: could they be related? Graefes Arch Clin Exp Ophthalmol. 2007;245(4):600-605.

175. Taban M, Sharma MC, Lee MS. Anterior ischemic optic neuropathy after uncomplicated scleral buckling surgery. Graefes Arch Clin Exp Ophthalmol. 2006;244(10):1370-1372.

176. Arnold AC. Anterior ischemic optic neuropathy following ocular pneumoplethysmography. J Clin Neuroophthalmol. 1987;7(1):58-59.

177. Mahroo OA, Hammond CJ. Anterior ischemic optic neuropathy after strabismus surgery. J Neuroophthalmol. 2009;29(2):157-158.

178. Kordic H, Flammer J, Mironow A, Killer HE. Perioperative posterior ischemic optic neuropathy as a rare complication of blepharoplasty. Ophthalmologica. 2005;219(3):185-188. 
179. Luscavage LE, Volpe NJ, Liss R. Posterior ischemic optic neuropathy after uncomplicated cataract extraction. Am J Ophthalmol. 2001;132(3):408-409.

180. Nawa Y, Jaques JD, Miller NR, Palermo RA, Green WR. Bilateral posterior optic neuropathy after bilateral radical neck dissection and hypotension. Graefes Arch Clin Exp Ophthalmol. 1992;230(4):301-308.
181. Dodd FM. Blindness following bilateral neck dissection. Eur $J$ Anaesthesiol. 1993;10(1):37-39.

182. Pliam MB, Cohen M, Cheng L, Spaenle M, Bronstein MH, Atkin TW. Pituitary adenomas complicating cardiac surgery: summary and review of 11 cases. J Card Surg. 1995;10(2):125-132.

\section{Publish your work in this journal}

Clinical Ophthalmology is an international, peer-reviewed journal covering all subspecialties within ophthalmology. Key topics include: Optometry; Visual science; Pharmacology and drug therapy in eye diseases; Basic Sciences; Primary and Secondary eye care; Patient Safety and Quality of Care Improvements. This journal is indexed on

\section{Dovepress}

PubMed Central and CAS, and is the official journal of The Society of Clinical Ophthalmology (SCO). The manuscript management system is completely online and includes a very quick and fair peer-review system, which is all easy to use. Visit http://www.dovepress.com/ testimonials.php to read real quotes from published authors. 